\title{
Caractérisation des pâturages herbacés sahéliens à accès limité : Cas du Centre Secondaire de Multiplication de Bétail de Sayam (Diffa).
}

\author{
Idrissa Issoufa ${ }^{1 *}$, Morou boubé ${ }^{1}$, Abdoulaye Diouf ${ }^{1,2}$, Saley Karim ${ }^{1}$ et Ali Mahamane ${ }^{3,4}$. \\ 1Département Biologie Végétale, Faculté des Sciences et Techniques, Université Dan Dicko Dankoulodo de Maradi, \\ B.N. 465 Maradi, Niger \\ 2Département des Sciences du Sol, Faculté des Sciences de l'Environnementales, Université Dan Dicko Dankoulodo \\ de Maradi, \\ ${ }^{3}$ Département Biologie Végétale, Faculté des Sciences et Techniques, Université Abdou Moumouni de Niamey, B.P. \\ 10960 Niamey, Niger \\ 4 Université de Diffa, B.P. 78, Niger \\ *Auteur correspondant : E- mail issoufaidrissa2@gmail.com / issoufad@yahoo.fr \\ Original submitted in on $12^{\text {th }}$ October 2020. Published online at www.m.elewa.org/journals/ on 31st December 2020 \\ https://doi.org/10.35759/JABs.156.4
}

\section{RÉSUMÉ}

Objectifs : Cette étude vise à caractériser l'état actuel des pâturages herbacés du Centre Secondaire de Multiplication de Bétail de Sayam.

Méthodologie et Résultats : Quatre groupements pastoraux ont été identifiés et décrits à partir de 56 relevés linéaires réalisés sur un transect Sud - Nord. Au total 98 espèces herbacées réparties parmi 59 genres et 27 familles botaniques ont été répertoriées. Les genres suivants: Eragrostis, Ipomoea, Aristida, Cenchrus, Indigofera, Digitaria et Brachiaria ont été les mieux représentés. Ils représentent environ $50 \%$ du total et renferment l'essentiel des espèces de bonne valeur pastorale. Le recouvrement global, les valeurs pastorales brutes et nettes sont respectivement de $85,5 \%, 75,05 \%$ et $67,56 \%$, celles-ci attestent que le pâturage du centre est de bonne qualité. La productivité globale en phytomasse herbacée a été estimée 2,26 tMS/ha avec une capacité de charge globale de 0,40 UBT/ha/an soit 2,5 ha/UBT/an. L'analyse du spectre fourrager indique que les espèces de bonne valeur pastorale et de faible valeur pastorale dominent le tapis herbacé. La première catégorie domine la zone centrale mieux protégée tandis que la dernière occupe les parties périphériques soumises à une forte exploitation du bétail.

Conclusion et application des résultats : Le front de colonisation des espèces faiblement appétées (espèces qui ont IS = 0 ou IS =1) progresse vers le centre sous l'effet de la forte pression pastorale. D'où l'urgence de clôturer l'ensemble du périmètre du centre afin d'éviter de créer un déséquilibre écologique préjudiciable à la survie de nombreuses espèces inféodées à ces milieux particuliers. Dans les perspectives d'une gestion durable des ressources fourragères des centres secondaire de multiplication de bétail, ce travail fournit des informations scientifiques fiables sur l'état actuel des pâturages herbacés de ce centre.

Mots-clés : Kouri, composition floristique, groupements pastoraux, qualité pastorale, Sayam. 
Characterization of Sahelian herbaceous pastures with limited access: Case of the Secondary Cattle Multiplication Center of Sayam (Diffa).

\begin{abstract}
Objectives: This study aims to characterize the current state of the herbaceous pastures of the Sayam Secondary Cattle Multiplication Center.

Methodology and Results : Four pastoral groups have been identified and described from 56 linear surveys carried out on a South - North transect. A total of 98 herbaceous species distributed among 59 genera and 27 botanical families have been listed. The best represented genera are: Eragrostis, Ipomoea, Aristida, Cenchrus, Indigofera, Digitaria and Brachiaria. They represent about $50 \%$ of the total and contain most of the species of good pastoral value. The overall recovery, the gross and net pastoral values are respectively $85.5 \%, 75.05 \%$ and $67.56 \%$, these attest that the pasture of the center is of good quality. The overall productivity of herbaceous phytomass was estimated at $2.26 \mathrm{tMS} /$ ha with an overall carrying capacity of $0.40 \mathrm{TLU} /$ ha / year, ie 2.5 ha / TLU / year. Analysis of the forage spectrum indicates that species of good pastoral value and those of low pastoral value dominate the grass cover. The first category dominates the better protected central zone while the last occupies the peripheral parts subject to heavy livestock exploitation.

Conclusion and applicability of results: The colonization front of poorly palatable species is progressing towards the center under the effect of strong pastoral pressure. Hence the urgency to enclose the entire perimeter of the center in order to avoid creating an ecological imbalance detrimental to the survival of many species dependent on these particular miles. With a view to the rehabilitation and sustainable management of these pastoral centers, this study provides additional information on the current state of the herbaceous pastures in this center.
\end{abstract}

Keywords : Kouri, floristic composition, pastoral groups, pastoral quality, Sayam, Niger

\section{INTRODUCTION}

Les pâturages naturels sahéliens jouent un rôle indispensable dans l'alimentation du bétail ; ils constituent la base et le plus souvent la totalité des ressources alimentaires des ruminants en élevage extensif voir même semi-intensif (Agonyissa et Sinsin, 1998). Selon les mêmes auteurs, les bovins tirent plus de $90 \%$ de leur énergie des pâturages naturels. Le Niger, est un pays sahélien à vocation essentiellement agropastorale en raison de ses immenses ressources animales. En effet, le cheptel national est estimé à plus 37 millions de têtes, pour une valeur monétaire de plus de 2000 milliards de F CFA (République du Niger, 2013). Ce cheptel tire l'essentiel de son alimentation des vastes étendues des pâturages naturels estimés à 62 millions d'hectares, soit plus de la moitié du pays (Rhissa, 2010). L'élevage et l'agriculture constituent les principales activités économiques des populations du Niger notamment celles du monde rural. II représente la source de revenu essentiel, souvent unique pour ces populations et un élément clé du commerce extérieur. En effet, il vient juste après les ressources minières (uranium et pétrole) en termes de recettes d'exportation et contribue à hauteur de $11 \%$ au PIB national, $40 \%$ au PIB agricole et $25 \%$ du budget des ménages. Cette forte contribution fait de ce secteur une arme efficace dans l'inlassable lutte contre la pauvreté et l'insécurité alimentaire (République du Niger, 2013). Cependant, le système d'élevage nigérien reste très traditionnel, sauf au niveau des ranchs ou centres secondaires aménagés par l'État où il est de type semi-intensif. La création de ces centres remonte de la période coloniale. Le premier ranch fut créé en 1931 à Toukounous avec pour mission principale la sélection et la diffusion du zébu Azawak dans les élevages traditionnels (Saidou et al., 2013). Après l'indépendance, d'autres Centres Secondaires de Multiplication de Bétail (CSMB) furent installés parmi lesquels figure celui de Sayam crée en 1976. Ce dernier avait pour mission principale la sélection, la diffusion et la conservation de la race Kouri. Cette 
dernière est unique en son genre et possède des qualités intéressantes notamment, des performances laitières, bouchères et surtout son adaptation au milieu (Adamou et al., 2018). L'élevage du bovin Kouri procure à ceux qui le pratiquent, du lait, de la viande, de l'argent et représente ainsi, une contribution essentielle aux moyens d'existence et à la sécurité alimentaire des ménages (Barma, 2017). Ces différentes potentialités cadrent parfaitement avec les enjeux des productions animales au Niger. Cependant, malgré ses atouts et sa place importante dans l'économie familiale, elle a été déclarée officiellement par la FAO (2004), pool génétique unique de l'Afrique en voie de disparition. Plusieurs facteurs expliquent la réduction des effectifs parmi lesquels figurent les déficits fourragers chroniques consécutifs aux récurrents chocs climatiques. Des études ont été effectuées au niveau du Sayam et ont concerné essentiellement la caractérisation du taurin kouri. Toutefois, on note au passage les travaux du pastoraliste Peyre de Fabrègues dans

\section{MATÉRIEL ET MÉTHODES}

Site d'étude : La présente étude a été réalisée dans le Centre Secondaire de Multiplication de Bétail (CSMB) de Sayam, situé dans le département de Diffa à $70 \mathrm{~km}$ au Nord-Est du chef-lieu de la Région (figure 1). II a été créé en 1976 avec un effectif de 13 bovins à dominance zébus Bororo d'une superficie de 29190,70 ha et de 70,5 $\mathrm{km}$ de périmètre. II compte en 2017 un effectif de 918 têtes de bovins Kouri. Le CSMB est établi sur des formations dunaires du continental terminal. Les sols sont sableux dans leur majorité. Le climat est de type sahélien dans la partie sud et sahélo-saharien plus au nord (CIRAD, 1996). II est marqué par l'alternance de deux saisons : une longue saison sèche d'octobre à juin et une courte saison des pluies avec des précipitations courtes et souvent violentes entre juillet et août (Bodart et al., 2010). La pluviométrie moyenne enregistrée à la station synoptique de Sayam est de $256 \pm 96 \mathrm{~mm}$ (1997 à 2017). Elle est répartie sur trois mois (juillet, août et septembre). Cette pluviosité est plus concentrée au mois d'août. Au cours de ces deux décennies, environ $60 \%$ des années présentent des précipitations inférieures à la moyenne interannuelle (figure 2). Ces années sont dites les années 1960/70 au nord de Gouré et à Sayam ayant décrit sommairement les pâturages de la zone. Depuis lors, très peu d'études se sont focalisées sur cette zone. Ainsi, se pose un problème de disponibilité de données quantitatives et qualitatives du potentiel productif de cet écosystème particulier. Or, une meilleure connaissance du ratio entre les besoins alimentaires et les potentialités que regorgent ces écosystèmes pastoraux, permettent de gérer durablement les ressources fourragères disponibles, facteur essentiel de la survie, de la productivité et de la conservation de cette race en voie d'extinction. L'objectif global de l'étude est de connaitre la qualité pastorale et la distribution spatiale des ressources herbagères de la station. Plus spécifiquement il s'agit de :

- Déterminer le cortège floristique du centre ;

- $\quad$ Définir les divers types de pâturage ;

- $\quad$ Calculer : la valeur pastorale, la productivité et la capacité de charge.

sèches. Cette zone se caractérise par l'irrégularité spatiale des pluies. Les températures moyennes mensuelles minimale et maximale sont respectivement de $14^{\circ} \mathrm{C}$ (janvier) à $43,5^{\circ} \mathrm{C}$ (mai) et peut atteindre $50^{\circ} \mathrm{C}$ à l'ombre. La végétation de la zone d'étude appartient au compartiment nord-sahélien oriental et sud saharien oriental (Saadou, 1990). La composante ligneuse forme une steppe arborée à Acacia tortilis (Forsk.) et Balanites aegyptiaca (L.) Del. et arbustive (Leptadenia pyrotechnica (Forsk.) Decne. et Acacia senegal (L.) Willd. Et le tapis herbacé est constituée principalement d'espèces graminéennes annuelles (formations à Aristideae). Cette station abrite également une diversité faunistique très importante dont certaines espèces sont en danger critique d'extinction en raison d'une part d'un braconnage permanent et d'autre part la dégradation continue de leurs habitats. Parmi les espèces fauniques rencontrées sur ce site figurent de nombreux oiseaux (Outardes arabe et Nubie, Falco biarmicus, Bubulcus ibis), des mammifères (Gazelle dama, Vulpes zerda), des reptiles (Varanus griseus, Serpent des sables, Chamaeleo africanus). 


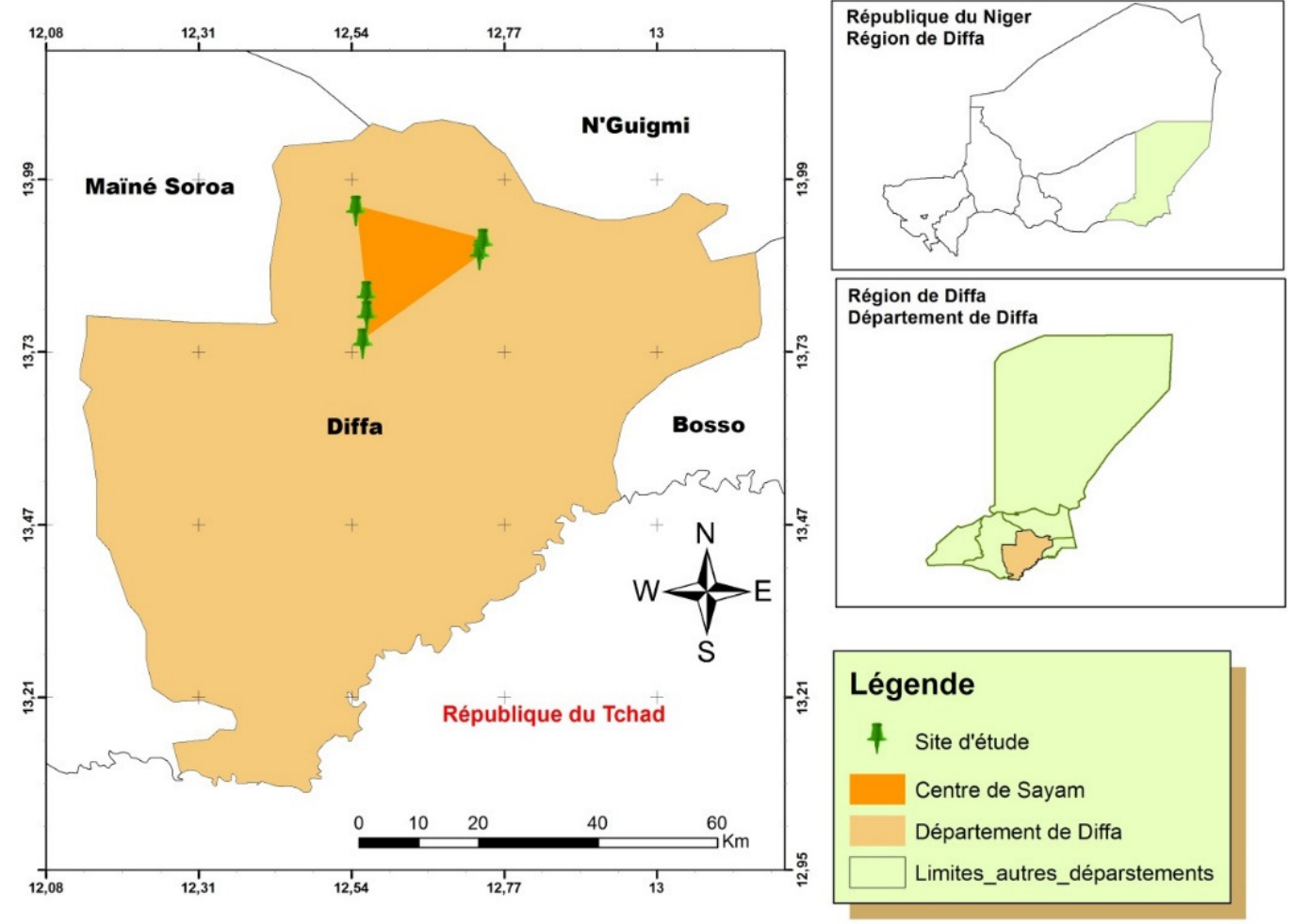

Figure 1 : localisation de site d'étude.

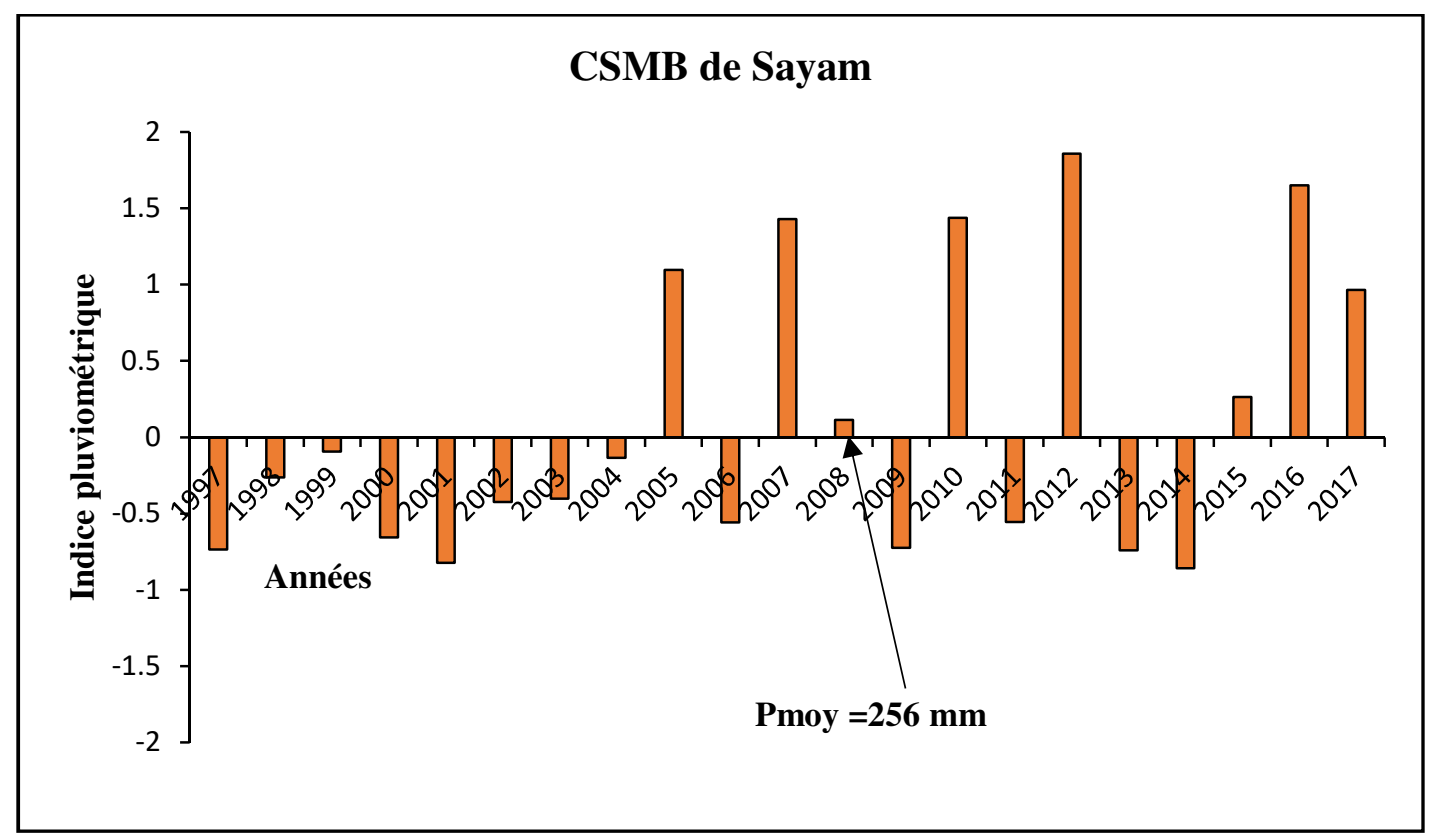

Figure 2 : Analyse des tendances évolutives de la pluviométrie annuelle par la méthode moyennes mobiles pondérées.

\section{Méthodes}

Échantillonnage: Dans le cadre de ce travail, un échantillonnage systématique le long d'un transect qui va du Sud (à l'entrée de la station) au Nord (limite de la station) a été appliqué. Les relevés floristiques ont été effectués suivant les unités géomorphologiques. Un total de 56 relevés de $2500 \mathrm{~m}^{2}(50 \mathrm{~m} \times 50 \mathrm{~m}$ ) équidistants de $500 \mathrm{~m}$ a été effectué pendant la période de maturation des espèces herbacées (Août et Septembre 2017). L'emplacement de relevés tient compte de 
l'homogénéité de la végétation herbacée, ceci pour éviter l'effet de lisière (Mahamane, 2005).

Collecte des données: L'inventaire des pâturages herbacés a été effectué selon la méthode des points quadrats alignés de Daget et Poissonnet (1971). Elle a consisté à tendre une cordelette graduée entre deux piquets au-dessus du tapis herbacée. Quatre lignes parallèles et distantes ont été dressées avec 50 points de lecture par ligne. L'enregistrement des espèces présentes a été effectué à l'aide d'une tige métallique enfoncée tous les $10 \mathrm{~cm}$ dans la végétation herbacée perpendiculairement à la ligne. La première ligne a été placée à cinq mètres de la bordure pour éviter l'effet de celle-ci (Alhassane, 2019). Les paramètres stationnels ont été notés dans chaque placette: il s'agit des coordonnées géographiques, la topographie et le type de sols a été apprécié par la méthode tactile. Des échantillons botaniques sont présentés tous les soirs aux bergers (10) disposant d'une connaissance empirique des espèces herbacées fourragères, cela a permis d'appréhender leur degré d'appréciation de l'appétence des espèces herbagères. En effet, en l'absence d'analyse chimique, la qualité d'un herbage est déterminée par le calcul de la valeur pastorale (Akpo et al., 2003). À chaque espèce herbacée un indice de qualité spécifique (IS) a été attribué (Delpech, 1960 ; Daget et Poissonnet, 1990; Soumana, 2011). Les indices de certaines espèces ont été obtenus à l'aide des recherches bibliographiques. Le classement des espèces a été fait suivant une échelle de cotation de 0 à 3 (Alhassane et al., 2018 ; Ngom et al., 2012 ; Akpo et al., Akpo et Grouzis, 2000 et 2002 ; Barral et al., 1983), c'est-à-dire sur une échelle de quatre classes $(0,1,2$ et 3) (Tableau 1). Certaines espèces ont été déterminées surplace, d'autres au laboratoire à travers la flore du Sénégal (Berhaut, 1967) et les Poaceae du Niger (Poilecot, 1999). La phytomasse a été évaluée via les techniques de la récolte intégrale, estimée particulièrement fiable (Yoka et al., 2010). Elle a consisté à couper toute la matière végétale espèce par espèce à l'intérieur de cinq carrés de biomasse de $1 \mathrm{~m}^{2}$ chacun dont quatre aux angles de la placette et un au centre. Cela permet d'évaluer la contribution de chaque espèce dans la biomasse. La biomasse récoltée a été ensachée puis pesés au frais à l'aide d'un peson. Cette biomasse a été séchée uniquement au soleil, puis transportés au laboratoire et pesé à nouveau pour déterminer le taux de la matière sèche.

Tableau1 : échelle d'intérêt pastoral des espèces herabagères établies avec les bergers du CSMB

\begin{tabular}{|l|l|l|}
\hline $\begin{array}{l}\text { Classe d'indice de qualité } \\
\text { spécifique }\end{array}$ & $\begin{array}{l}\text { Appréciation de la qualité } \\
\text { fourragère }\end{array}$ & $\begin{array}{l}\text { niveau d'appétence par les } \\
\text { bovins }\end{array}$ \\
\hline 0 & $\begin{array}{l}\text { sans valeur pastorale (Svp) } \\
\text { faible valeur pastorale (Fvp) }\end{array}$ & non appété \\
1 & peu appété \\
2 & moyenne valeur pastorale (Mvp) & moyennement appété \\
3 & bonne valeur pastorale (Bvp) & très bien appété \\
\hline
\end{tabular}

Traitement des données : Les données collectées sur les fiches de relevés floristiques ont servi de base pour la caractérisation de la flore herbacée du centre. Elles ont été saisies et traitées à partir du tableur Excel. Plusieurs descripteurs phytoécologiques ont été déterminés.

Indices de diversité et de régularité : L'indice de diversité de Shannon-Weaver ( $\left.H^{\prime}\right)$ a été utilisé pour analyser la distribution des espèces végétales dans l'espace avec la formule suivante:

\section{$H^{\prime}=-\Sigma p i \log 2 p i$}

$H^{\prime}=$ indice de diversité de Shannon, $p i=$ poids de l'espèce $i$ dans le groupement; $p i=$ ni/ $\Sigma$ ni, avec ni $=$ recouvrement moyen de l'espèce $i$ et $\Sigma \mathrm{ni}=$ recouvrement moyen total de toutes les espèces. Son unité est bit. La régularité de Pielou $(\mathrm{E})$ apprécie le poids de chaque espèce dans l'occupation de l'espace. Sa formule est par:

$$
E=\frac{H^{\prime}}{\log 2 S}
$$

Avec $\mathrm{E}=$ équitabilité de Pielou, $S=$ nombre total d'espèces consécutives du groupement, log2 = diversité maximale de Shannon et varie de 0 à 1 . La similitude entre deux groupements étudiés a été calculée par le coefficient de similitude de Sorensen(I) (1948). Son expression est la suivante :

$$
I=(2 C / A+B-C) \times 100
$$

$A=$ nombre d'espèces du groupement $1, B=$ nombre d'espèces du groupement $2, C=$ nombre total d'espèces communes aux deux groupements. Le logiciel CAP a servi pour son calcul.

Fréquence spécifique $(\mathrm{Fs})$ : La fréquence d'une espèce correspond à la somme des contacts de cette espèce. Elle a été calculée pour chaque espèce herbacée productive avec la formule suivante. 


$$
F S i(\%)=n i \times \frac{100}{N}
$$

$\mathrm{ni}=$ nombre de fois où l'espèce $\mathrm{i}$ a été recensée et $\mathrm{N}=$ l'ensemble des points échantillonnés.

Contribution spécifique : La contribution spécifique (Csi) d'une espèce, est le rapport, en pour cent, entre la Fs de cette espèce et la somme des fréquences spécifiques de toutes les espèces considérées. Elle est exprimée par la formule suivante : $\mathrm{CSi}=F S i \times \frac{\mathbf{1 0 0}}{\mathbf{E F S i}}$

Fsi = fréquence spécifique

$\Sigma F s i=$ somme des fréquences spécifiques de toutes les espèces recensées

Recouvrement global (RG) : II s'exprime par : RG $(\%)=((\mathrm{N}-\mathrm{n}) / \mathrm{N}) * 100$

$\mathrm{N}$ est le nombre total de contact ; $n$ est nombre de points où on trouve des plages du sol nu.

Le recouvrement spécifique est le rapport entre la fréquence spécifique de l'espèce (i) sur le nombre total des points de lecture du relevé. Rs $(\%)=\mathrm{Fsi} / \mathrm{N}$.

$\mathrm{Rs}=$ recouvrement spécifique; $\mathrm{Fs}=$ fréquence spécifique et $\mathrm{N}=$ nombre total de points de lecture du relevé.

Valeur pastorale : La valeur pastorale brute $(\mathrm{Vpb})$ de chaque groupement a été appréciée par : Vpb $=1 / 3 \times \Sigma C$ Cix $\mid$ si. Elle est en pourcent $(\%)$ et fluctue entre 0 et $100 \%$. $\mathrm{Vpb}=$ valeur pastorale brute. La valeur pastorale tient compte de la période de l'appétibilité de la plante, le degré d'appétibilité lié à l'anatomie et à la morphologie des feuilles et des tiges et de la valeur fourragère (Ngom et al., 2012). Pour éviter le problème de surestimation, la valeur pastorale a été pondérée par le recouvrement global de la végétation (RGV) de chaque type de pâturage, comme cela a été recommandé par les chercheurs (Ngom et al., 2012; Akpo et Grouzis, 2000 et Aidoud, 1983). La valeur pastorale nette, ou indice global de qualité se calcule par : $V p n=I G Q=R G V \times 0,3 \Sigma C S i \times \mid s i$. Avec $V p n=$ valeur pastorale nette, $I G Q=$ indice global de qualité, $R G V=$ recouvrement global de la végétation, IS = indice spécifique. Selon Ngom et al. (2012), la connaissance de $V p n$ et de la production de fourrage "qualifié »

\section{RESULTATS}

Analyse globale de la composition botanique: L'inventaire des pâturages herbacés du CSMB a permis de recenser 98 espèces (Tableau 2), réparties parmi 59 genres, appartenant à 27 familles botaniques d'importance variable. L'analyse de la figure 3 montre que la famille des Poaceae est la mieux représentée avec plus du tiers des espèces (33 espèces, 33,67\%), permet de mieux appréhender la durabilité d'un écosystème pastoral. Pour calculer la production de fourrage qualifié (Pfq) (Ngom et al., 2012 ; Akpo et al., 2002) du centre, IGQ a été appliqué à la biomasse (Ph). $P f q=P h \times I G Q ; P f q=$ production de fourrage qualifié, $\mathrm{Ph}=$ phytomasse herbacée.

Capacité de charge : La capacité de charge (CC) se définit par le nombre d'Unité Bétail Tropical (UBT, animal de référence avec poids vif $=250 \mathrm{~kg}$ ) qu'on peut y faire vivre de manière durable sans se détériorer (Baumer, 1997). On part du postulat qu'une UBT a un besoin alimentaire quotidien équivalent à $6,25 \mathrm{~kg}$ de matière sèche. Elle s'exprime par: CC (UBT/ha/an) = (productivité $(\mathrm{kg} \mathrm{Ms} / \mathrm{ha}) \times \mathrm{U} /(6,25 \times$ période d'utilisation (270 jours)). Avec Productivité (kgMs/ha): c'est la productivité moyenne annuelle en phytomasse (Ms) du pâturage ; $U$ : correspond au coefficient d'utilisation potentielle de la biomasse et l'on retient $1 / 3$ pendant la phase de maturation des graminées (Ouédraogo, 2009). Discrimination des groupements pastoraux: L'individualisation des groupements a été réalisée à partir des analyses multivariées. À cet effet, le croisement entre les tableaux de relevés-espèces et relevés-variables écologiques a été effectué au moyen de l'analyse canonique des correspondances (ACC) dans le Logiciel PC-ORD 5 (McCune et Grace, 2002). Cette mise en relation permet d'évaluer la proportion de la variabilité floristique pouvant être expliquée par les variables environnementales (Mahamane, 2005). Pour identifier les espèces qui contribuent significativement au maintien des groupements, celles-ci testées par Indicator Species Analysis (ISA) (Dufrêne et Legendre, 1997.) disponible sur le même logiciel. L'ISA combine la fréquence relative et l'abondance relative pour le calcul de la valeur indicatrice de chaque espèce dont la signification est appréciée par le test de Monte-Carlo. Toute espèce dont la probabilité $(P)$ inférieure à 0,05 a été considérée comme espèce caractéristique du groupement (Ouôba, 2006). Pour nommer les groupements, la combinaison de deux espèces ayant les plus grandes valeurs indicatrices et les plus faibles valeurs de $\mathrm{P}$ (probabilité) a été utilisée.

suivie de très loin de celles des Rubiaceae (8 espèces, $8,16 \%$ ), des Convolvulaceae (7 espèces, $7,14 \%$,) et des Fabaceae $(6,12 \%, 6$ espèces). Ces quatre premières familles ont rassemblé 54 espèces, soit $55 \%$ de l'effectif global. Douze (12) familles ont été représentées par une seule espèce soit $12,24 \%$ de l'ensemble des espèces inventoriées. En termes de genres: Eragrostis (6 
espèces), Ipomoea (5 espèces), Aristida, Cenchrus et Indigofera (4 espèces chacun), Digitaria et Brachiaria (3 espèces chacun) sont les mieux représentés. Ces genres renferment l'essentiel des espèces de bonne valeur pastorale. Quatre taxons dominent essentiellement le peuplement herbacé du CSMB avec des contributions spécifiques supérieures à $10 \%$. II s'agit de Cenchrus biflorus Roxb. (16,77\%), Chloris prieurii Kunth. (13,69\%), Tribulus terrestris L. (12,24\%) et Aristida mutabilis Trin. \& Rupr. (11,09\%). Trois de ces espèces présentent un bon intérêt pastoral $(I s=3)$ et Tribulus terrestris L. porte l'indice de qualité faible (Is = 1). Les contributions spécifiques de ces espèces représentent plus de $53 \%$ du total. L'on remarque l'absence quasi-totale de légumineuse Zornia glochidiata Reich. Ex DC. $(0,02 \%$ de Csi) dans le tapis herbacé.

Types biologiques et phytogéographiques: L'examen de la figure 4 du spectre des types biologiques montre la prépondérance des thérophytes par rapport aux autres formes de vie (86 espèces; $72,23 \%$ du spectre brut et $61,4 \%$ du spectre pondéré). Ils sont suivis de très loin par les microphanérophytes (12 espèces ; $10,1 \%$ du spectre brut et $32,1 \%$ du spectre pondéré). Les lianes chaméphytes sont les moins représentées avec une seule espèce soit $0,8 \%$ du total. Les thérophytes ont donc imprimé la physionomie du pâturage herbacé du ranch de Sayam. L'analyse du spectre des types phytogéographiques (figure 5) révèle que la flore herbacée est principalement dominée par les espèces à large distribution (66 espèces ; $55,7 \%$ du spectre brut et $68,7 \%$ du spectre pondéré). Elles sont suivies par les espèces soudano-zambéziennes (14 espèces ; $11,8 \%$ du spectre brut et $10,4 \%$ du spectre pondéré). Les types phytogéographiques les moins représentés n'ont qu'une seule espèce. La prééminence des espèces Paléotropicales et Pantropicales dénote que le tapis herbacé est couvert essentiellement par des espèces étrangères.

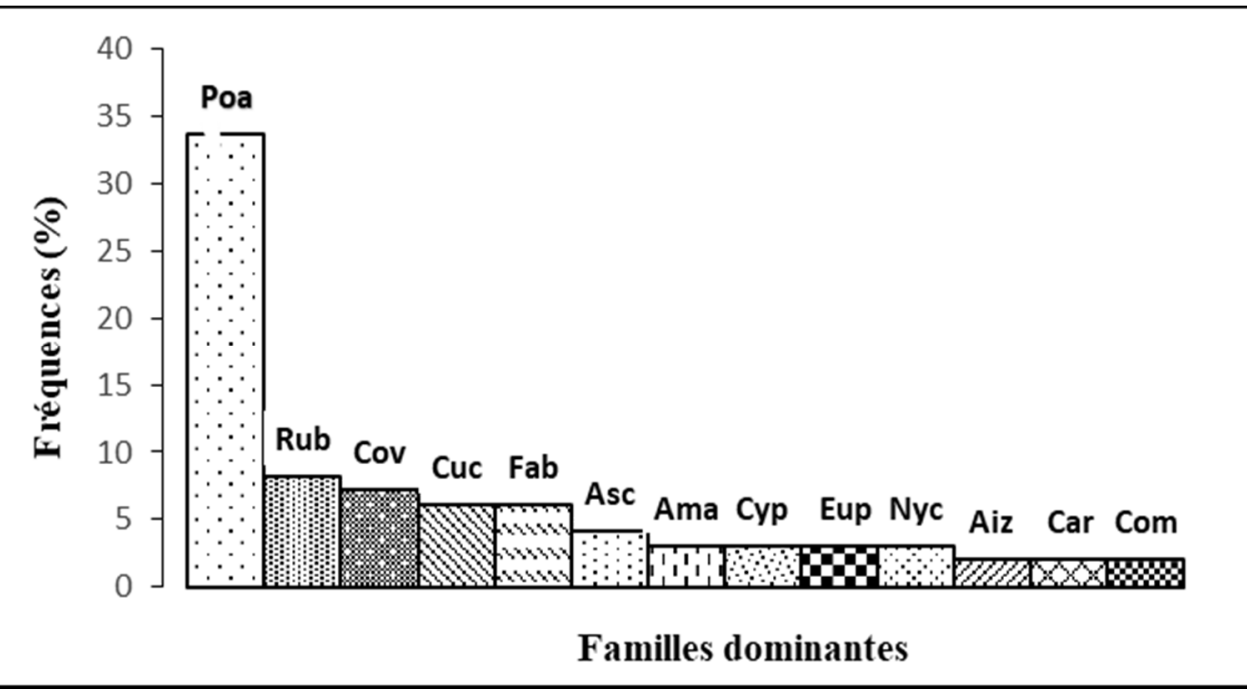

Figure 3 : Spectre des familles dominantes au niveau du CSMB.

Poa : Poaceae, Rub : Rubiaceae, Cov : Convolvulaceae, Fab : Fabaceae, Asc : Asclepiadaceae, Ama : amranthaceae, Cyp : Cyperaceae, Eup : Euphorbiaceae, Aiz : Aizoaceae, Car : Caryophyllaceae et Com : Commelinaceae 
Idrissa et al., J. Appl. Biosci. 2020 Caractérisation des pâturages herbacés sahéliens à accès limité : Cas du Centre Secondaire de Multiplication de Bétail de Sayam (Diffa).

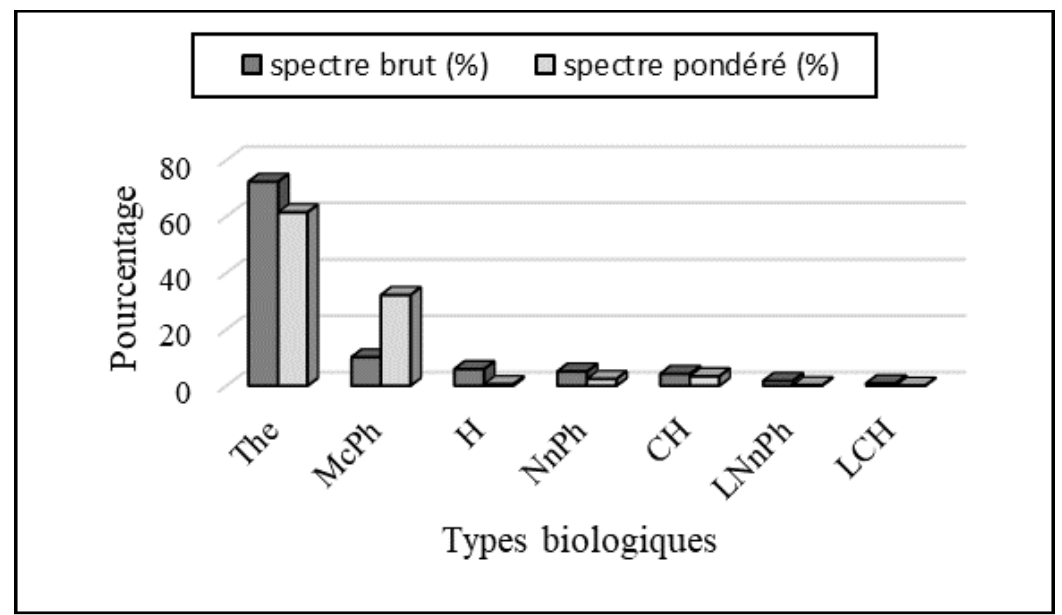

Figure 4 : spectre des types biologiques.

The : thérophytes, $\mathrm{McPh}$ : microphanérophytes, $\mathrm{H}$ : hémicryptophytes, $\mathrm{NnPh}$ : nanophanérophytes, $\mathrm{CH}$ : chaméphytes, $\mathrm{LNnPh}$ : liane nanerophytes et LCH : lianes chaméphytes.

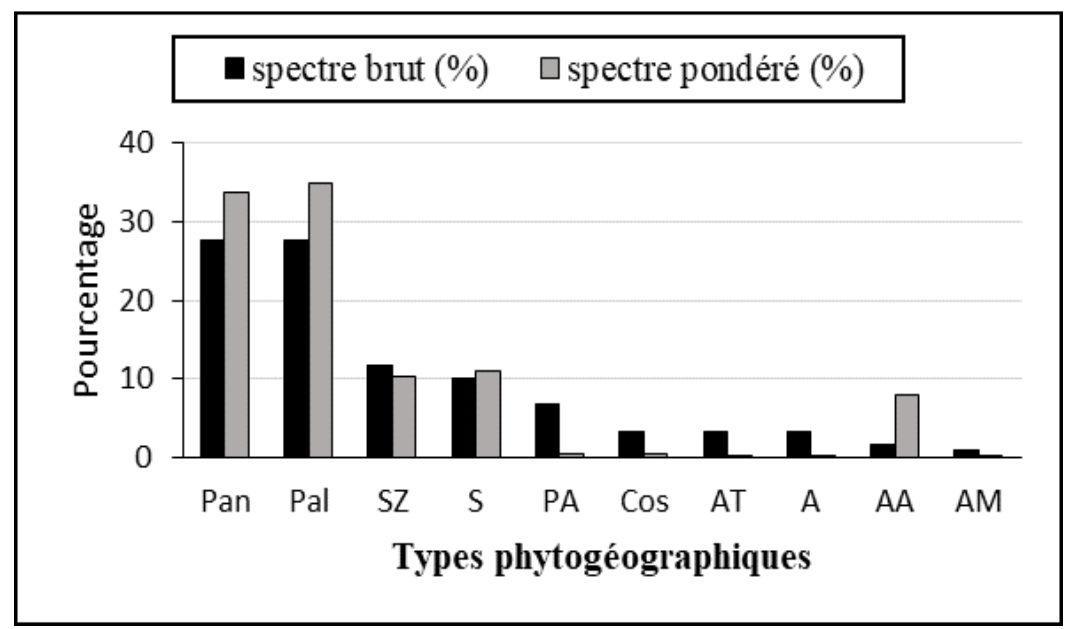

Figure 5 : spectre des types phytogéographiques.

Pan : espèces pantropicales, Pal : espèces paléotropicales, $S Z$ : espèces soudano- zambéziennes, $S$ : élément- base soudanien, Cos : espèces cosmopolites, AT : espèces afro-tropicales, A : africaines, AA : espèces afro- américaines, AM : afro-malgaches.

Valeur pastorale: Les valeurs pastorales relatives (Vpr) des espèces herbacées ont fluctué des moyennes à nulles (Tableau 2). Les espèces telles que Cenchrus biflorus Roxb. $(50,32)$, Chloris prieurii Kunth. $(41,06)$, Aristida mutabilis Trin. \& Rupr. $(33,28)$, Schoenefeldia gracilis Kunth. $(20,06)$ Dactyloctenium aegyptium (L.) Willd. $(15,57)$ ont présenté les valeurs pastorales relatives les plus élevées. Dans la strate herbacée du CSMB, la diversité spécifique des catégories fourragères et le nombre d'espèce par catégorie ont été variables. Ainsi, l'examen du spectre fourrager de ces catégories (figure 6) a montré que les espèces de bonne valeur pastorale (24 espèces, soit $63 \%$ de Csi) ont été partout les plus importantes comparées aux autres catégories fourragères. Elles sont suivies de loin par celles de faible valeur pastorale (29 espèces, $25 \%$ de Csi). Les contributions spécifiques de ces deux catégories représentent $88 \%$ du total. Les plantes totalement délaissées (Svp : 4,25\% de Csi) et celles de moyenne valeur pastorale (Mvp : $7 \%$ de Csi) ont été globalement très rares. Elles ont donc faiblement contribué aux valeurs pastorales du fait leurs faibles contributions spécifiques. Les valeurs pastorales brute et nette de la station sahélienne ont été respectivement de $75,05 \%$ et $67,56 \%$. 


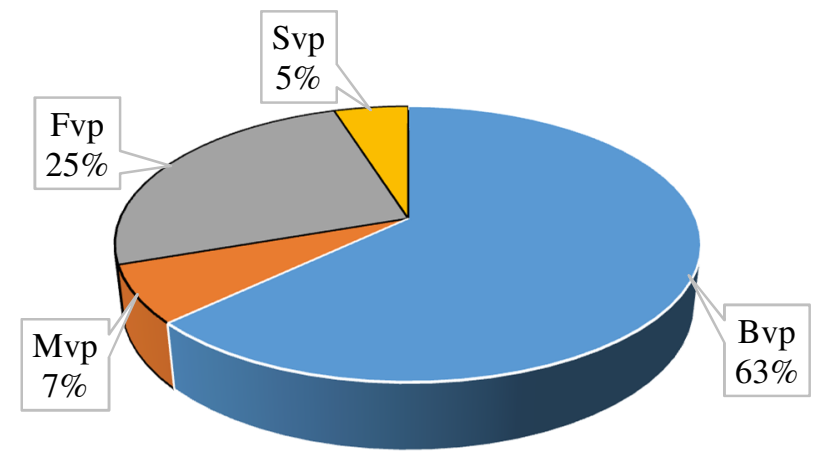

Figure 6 : spectre des catégories fourragères.

Bvp : bonne valeur pastorale, Mvp : moyenne valeur pastorale Fvp : faible valeur pastorale et Svp : sans valeur pastorale.

Tableau 2 : Composition floristique, fréquence spécifique, recouvrement et contribution spécifique.

\begin{tabular}{|c|c|c|c|c|c|c|c|c|}
\hline Familles & TB & TP & Genre et espèce & \begin{tabular}{|l|}
$\mathrm{FSi}$ \\
$(\%)$
\end{tabular} & \begin{tabular}{|l|} 
Rs \\
$(\%)$
\end{tabular} & $\begin{array}{l}\text { CSC } \\
(\%)\end{array}$ & IS & \begin{tabular}{|l}
$\mathrm{Vr}=$ \\
CSC ${ }^{*}$ IS
\end{tabular} \\
\hline Poaceae & The & Pal & Cenchrus biflorus Roxb. & 30,5 & 15,25 & 16,77 & 3 & 50,32 \\
\hline Poaceae & The & AA & Chloris prieurii Kunth. & 24,88 & 12,44 & 13,69 & 3 & 41,06 \\
\hline Zygophyllaceae & The & Pan & Tribulus terrestris $L$. & 22,25 & 11,13 & 12,24 & 1 & 12,24 \\
\hline Poaceae & The & Pan & Aristida mutabilis Trin. \& Rupr. & 20,17 & 10,08 & 11,09 & 3 & 33,28 \\
\hline Poaceae & The & Pal & Schenefeldia gracilis Kunth. & 12,16 & 60,8 & 6,69 & 3 & 20,06 \\
\hline Fabaceae & The & Pan & Alysicarpus ovalifolius (Schum. \& Thonn.). & 11,18 & 5,59 & 6,15 & 2 & 12,3 \\
\hline Poaceae & The & Pal & Dactyloctenium aegyptium (L.) Willd. & 9,44 & 4,07 & 5,19 & 3 & 15,57 \\
\hline Cyperaceae & The & Pan & Cyperus amabilis vahl. & 7,37 & 3,68 & 4,05 & 1 & 4,05 \\
\hline Poaceae & The & SZ & Brachiaria xantholeuca (Schinz.) Stapf. & 7,19 & 3,59 & 3,95 & 3 & 11,86 \\
\hline Tiliaceae & The & Pal & Corchorus tridens $L$. & 6,73 & 3,33 & 3,7 & 1 & 3,7 \\
\hline Poaceae & The & A & Panicum anabaptistum Steud. & 4,09 & 2,04 & 2,25 & 1 & 2,25 \\
\hline Poaceae & The & Cos & Eragrostis cilianensis (All.) F. T. Hubb. & 2,64 & 1,32 & 1,45 & 3 & 4,36 \\
\hline Caryophyllaceae & The & PA & Polycarpaea linearifolia (DC.) DC. & 0,48 & 1,29 & 1,42 & 0 & 0 \\
\hline Poaceae & $\mathrm{H}$ & SZ & Pennisetum violaceum (Lam.) L. Rich. & 2,31 & 1,16 & 1,27 & 3 & 3,82 \\
\hline Poaceae & The & SZ & Digitaria argillacea (Hitch. Et Chase) Fern. & 2,08 & 1,04 & 1,14 & 3 & 3,43 \\
\hline Boraginaceae & $\mathrm{CH}$ & Pan & Heliotropuim strigosum Willd. et H. Rariflorum & 0,13 & 0,68 & 0,75 & 0 & 0 \\
\hline Nyctaginaceae & The & Pan & Boerhavia repens $L$. & 1,26 & 0,63 & 0,69 & 1 & 0,69 \\
\hline Euphorbiaceae & $\mathrm{NnPh}$ & Pan & Chrozophora brochiana (Lam.) A. Juss. & 1,04 & 0,52 & 0,57 & 1 & 0,57 \\
\hline Rubiaceae & $\mathrm{CH}$ & Pal & Sida ovata Forsk. & 0,21 & 0,49 & 0,54 & 0 & 0 \\
\hline Poaceae & The & Pan & Tragus berteroniamus Schlt. & 0,85 & 0,42 & 0,47 & 2 & 0,93 \\
\hline Cyperaceae & The & S & Fimbritilyste huspidula (Valh.)Kunth. & 0,15 & 0,39 & 0,43 & 0 & 0 \\
\hline Amaranthaceae & The & Pan & Achyrentes aspera $L$. & 0,72 & 0,36 & 0,4 & 1 & 0,4 \\
\hline Poaceae & The & Pan & Aristida funiculata Trin. \& Rupr. & 0,56 & 0,28 & 0,31 & 3 & 0,93 \\
\hline Sterculiaceae & $\mathrm{NnPh}$ & Pan & Waltheria indica $L$. & 0,16 & 0,27 & 0,29 & 0 & 0 \\
\hline Poaceae & The & A & Brachiaria villosa (Lam.) A. Camus. & 0,97 & 0,24 & 0,27 & 3 & 0,8 \\
\hline Amaranthaceae & The & Cos & Amaranthus spinosus $L$. & 0,48 & 0,24 & 0,27 & 1 & 0,27 \\
\hline Rubiaceae & The & A & Kohautia senegalensis (Valh.)Kunth. & 0,3 & 0,15 & 0,17 & 1 & 0,17 \\
\hline Pedaliaceae & The & PA & Cerathoteca sesamoides End. & 0,09 & 0,15 & 0,16 & 0 & 0 \\
\hline Poaceae & The & Pal & Cenchus cialiaris $L$. & 0,28 & 0,14 & 0,15 & 3 & 0,46 \\
\hline Poaceae & $\mathrm{H}$ & Pal & Eragrostis tenella (L.) P. de Beauv. & 0,26 & 0,13 & 0,14 & 3 & 0,43 \\
\hline Rubiaceae & The & Pal & Oldendelia herbaceae (L.) Roxb. & 0,18 & 0,11 & 0,12 & 0 & 0 \\
\hline Convolvulaceae & The & Pal & Ipomoea coptica (L.) Roth. & 0,2 & 0,1 & 0,11 & 2 & 0,22 \\
\hline Aizoaceae & The & SZ & Gisekia phernacioides $L$. & 0,2 & 0,1 & 0,11 & 1 & 0,11 \\
\hline Pedaliaceae & The & SZ & Sesamum alatum Thonn. & 0,2 & 0,1 & 0,11 & 1 & 0,11 \\
\hline Rubiaceae & The & Pan & Polycarpaea eriantha Hochst. Ex A. Rich. & 2,58 & 0,1 & 0,11 & 0 & 0 \\
\hline Convolvulaceae & The & Pal & Merreimia tridentata (L.) Hallier & 0,19 & 0,9 & 0,1 & 2 & 0,21 \\
\hline
\end{tabular}


Idrissa et al., J. Appl. Biosci. 2020 Caractérisation des pâturages herbacés sahéliens à accès limité : Cas du Centre Secondaire de Multiplication de Bétail de Sayam (Diffa).

\begin{tabular}{|c|c|c|c|c|c|c|c|c|}
\hline Cucurbitaceae & The & Pan & Cucumis melo Naud. & 0,19 & 0,9 & $\mid 0,1$ & $\mid 1$ & $\mid 0,1$ \\
\hline Rubiaceae & The & Pan & Oldendelia corymbosa L. & 0,04 & 0,9 & 0,1 & 0 & 0 \\
\hline Poaceae & $\mathrm{H}$ & Pan & Cymbogon schoenantus (L.) Spreng & 0,17 & 0,08 & 0,09 & 2 & 0,19 \\
\hline Burseraceae & The & Pan & Commecarpus helenae A.Rich & 0,05 & 0,08 & 0,09 & 0 & 0 \\
\hline Malvaceae & $\mathrm{CH}$ & Pan & Sida cordifolia L. & 0,04 & 0,08 & 0,09 & 0 & 0 \\
\hline Commelinaceae & The & Pal & Commelina bengalensis $L$. & 0,15 & 0,07 & 0,08 & 2 & 0,17 \\
\hline Liliaceae & $\mathrm{Ge}$ & $S Z$ & $\begin{array}{l}\text { Dipcadi toccazeanum (Hochst. Ex A. Rich.) } \\
\text { Bak }\end{array}$ & 0,06 & 0,07 & 0,08 & 0 & 0 \\
\hline Poaceae & The & A & Eragrostis aegyptiaca Del. & 0,14 & 0,07 & 0,08 & 3 & 0,24 \\
\hline Euphorbiaceae & The & Pal & Phyllanthus pentendrus Schum. Thonn. & 0,14 & 0,07 & 0,08 & 2 & 0,16 \\
\hline Poaceae & The & Pan & Eragrostis tremula Steud. & 0,13 & 0,06 & 0,07 & 3 & 0,21 \\
\hline Poaceae & The & Pan & Aristida adscensionis $L$. & 0,13 & 0,06 & 0,07 & 2 & 0,14 \\
\hline Euphorbiaceae & The & Pan & Phyllanthus amarus Schum. \& Thon. & 0,04 & 0,06 & 0,07 & 0 & 0 \\
\hline Poaceae & The & Pan & Digitaria horozontalis Willd. & 0,12 & 0,05 & 0,06 & 3 & 0,19 \\
\hline Asteraceae & The & AT & Blainvillea gayana Cass. & 0,02 & 0,05 & 0,06 & 0 & 0 \\
\hline Poaceae & The & Pal & Indigofera hirsuta L. & 0,11 & 0,05 & 0,06 & 3 & 0,18 \\
\hline Fabaceae & $\mathrm{CH}$ & PA & Thephrosia purperea (L.) Pers. & 0,11 & 0,05 & 0,06 & 1 & 0,06 \\
\hline Poaceae & $\mathrm{H}$ & S & Andropogon gayanus Kunth. & 0,1 & 0,04 & 0,05 & 3 & 0,16 \\
\hline Convolvulaceae & The & Pan & Ipomoea kotschyana Hochst. Ex. Choisy. & 0,1 & 0,04 & 0,05 & 2 & 0,11 \\
\hline Poaceae & The & pal & Cenchrus pennisetiformis Steud. & 0,1 & 0,04 & 0,05 & 2 & 0,11 \\
\hline Poaceae & The & Pan & Digitaria ciliaris (Retz.) Koeler. & 0,1 & 0,04 & 0,05 & 2 & 0,11 \\
\hline Commelinaceae & The & Pal & Commelina forskoalei Vahl. & 0,09 & 0,04 & 0,05 & 2 & 0,1 \\
\hline Fabaceae & The & S & Indigofera senegalensis Lam. & 0,09 & 0,04 & 0,05 & 2 & 0,1 \\
\hline Poaceae & The & AT & Aristida stipoides Lam. & 0,09 & 0,04 & 0,05 & 2 & 0,1 \\
\hline Caryophyllaceae & $\mathrm{H}$ & SZ & Panicum anabaptistum Steud. & 0,09 & 0,04 & 0,05 & 1 & 0,05 \\
\hline Cyperaceae & $H$ & Pal & Cyperus conglomeratus Rottb. & 0,09 & 0,04 & 0,05 & 1 & 0,05 \\
\hline Laminaceae & The & Pan & Leucas martinicensis (Jacq.) R. Br. & 0,16 & 0,04 & 0,05 & 0 & 0 \\
\hline Cucurbitaceae & The & SZ & Cucumis metuliferus Naud. & 0,08 & 0,03 & 0,04 & 1 & 0,04 \\
\hline Convolvulaceae & The & S & Ipomea vagans Bak. & 0,07 & 0,03 & 0,04 & 3 & 0,12 \\
\hline Poaceae & The & Pal & Brachiaria ramosa (L.) Stapf. & 0,07 & 0,03 & 0,04 & 3 & 0,12 \\
\hline Poaceae & The & $A$ & Tephrosia bracteolata Guill. \& Perr. & 0,07 & 0,03 & 0,04 & 3 & 0,12 \\
\hline Fabaceae & The & S & Indigofera aspera Perr. & 0,07 & 0,03 & 0,04 & 2 & 0,08 \\
\hline Poaceae & The & Pal & Cenchrus setigesus Vahl. & 0,07 & 0,03 & 0,04 & 2 & 0,08 \\
\hline Polygalaceae & The & $A$ & Polygala multiflora Poir. & 0,07 & 0,03 & 0,04 & 2 & 0,08 \\
\hline Nyctaginaceae & The & $\cos$ & Boerhavia eractal L. & 0,06 & 0,02 & 0,03 & 1 & 0,03 \\
\hline Scrophulariaceae & The & AM & Striga hermontica (Del.) Benth. & 0,06 & 0,02 & 0,03 & 1 & 0,03 \\
\hline Asclepiadaceae & The & Pal & Cassia mimoides $L$. & 0,12 & 0,02 & 0,03 & 0 & 0 \\
\hline Convolvulaceae & The & $S Z$ & Ipomoea dichroa Hochst. ex Choisy. & 0,02 & 0,02 & 0,03 & 2 & 0,06 \\
\hline Cucurbitaceae & LCH & Pan & Citrilus colocynthis $L$. & 0,05 & 0,02 & 0,03 & 1 & 0,03 \\
\hline Cucurbitaceae & LNnPh & Pal & Cucumis prophetarum L. & 0,05 & 0,02 & 0,03 & 1 & 0,03 \\
\hline Nyctaginaceae & The & Cos & Amaranthus graecizans & 0,05 & 0,02 & 0,03 & 1 & 0,03 \\
\hline Poaceae & $\mathrm{H}$ & GC-SZ & Eragrostis atrovirens & 0,29 & 0,01 & 0,02 & 3 & 0,07 \\
\hline Convolvulaceae & The & AT & Merremia pinnata (Choisy.) $f$. & 0,04 & 0,01 & 0,02 & 2 & 0,05 \\
\hline Fabaceae & The & PA & Zornia glochidiata Reich. Ex DC. & 0,04 & 0,01 & 0,02 & 2 & 0,05 \\
\hline Capparidaceae & The & PA & Cloeme viscosa $L$. & 0,04 & 0,01 & 0,02 & 1 & 0,02 \\
\hline Rubiaceae & The & SZ & Spermacoce scabra (Schum. Et Thonn.) & 0,04 & 0,01 & 0,02 & 1 & 0,02 \\
\hline Amaranthaceae & $\mathrm{NnPh}$ & Pal & Aerva javanica (Burm.) Juss. ex Schult. & 0,54 & 0,01 & 0,02 & 0 & 0 \\
\hline Asclepiadaceae & The & Pan & Cassia obtusifolia L. & 1,37 & 0,01 & 0,02 & 0 & 0 \\
\hline Asclepiadaceae & $\mathrm{NnPh}$ & Pal & Pergularia tomantosa $L$. & 0,03 & 0,01 & 0,02 & 0 & 0 \\
\hline Cucurbitaceae & The & Pan & Momordica balsamina $L$. & 0,2 & 0,01 & 0,02 & 0 & 0 \\
\hline Convolvulaceae & The & Pal & Ipomea pes-tigridis $L$. & 0,04 & 0,01 & 0,02 & 2 & 0,04 \\
\hline Poaceae & The & $A$ & Loudetia hordeiformis (Stapf) Hubb. & 0,04 & 0,01 & 0,02 & 2 & 0,04 \\
\hline Malvaceae & The & PA & Hibiscus asper Hook. f. & 0,04 & 0,01 & 0,02 & 1 & 0,02 \\
\hline Rubiaceae & The & S & Spermacoce chaetocephala DC. & 0,04 & 0,01 & 0,02 & 1 & 0,02 \\
\hline Poaceae & The & Pan & Pennisetum pedicellatum & 0,03 & 0,01 & 0,01 & 3 & 0,04 \\
\hline Acanthaceae & The & Pal & Peristrophe bicalyculata (Retz.) Nees. & 0,03 & 0,01 & 0,01 & 1 & 0,01 \\
\hline
\end{tabular}


Idrissa et al., J. Appl. Biosci. 2020 Caractérisation des pâturages herbacés sahéliens à accès limité : Cas du Centre Secondaire de Multiplication de Bétail de Sayam (Diffa).

\begin{tabular}{l|l|l|l|l|l|l|l|l} 
Cucurbitaceae & The & PA & Citrilus lanatus (Thunb.) & 0,03 & 0,01 & 0,01 & 1 & 0,01 \\
Poaceae & The & S & Ctenium eleganse Kunth. & 0,03 & 0,01 & 0,01 & 1 & 0,01 \\
Rubiaceae & The & SZ & Spermacoce radiata (DC.) Hiern. & 0,03 & 0,01 & 0,01 & 1 & 0,01 \\
Aizoaceae & The & SZ & Mollugo nudicaulis Lam. & 0,79 & 0,01 & 0,01 & 0 & 0 \\
Poaceae & The & Pan & Chloris barbata Sw. & 0,02 & 0,01 & 0,01 & 3 & 0,03 \\
Poaceae & H & PA & Sporobolus festivus Hochst. ex A. Rich. & 0,04 & 0,01 & 0,01 & 0 & 0 \\
Fabaceae & The & Pal & Indigofera duphylla Vent. & 0,01 & 0,01 & 0 & 2 & 0,01 \\
\hline
\end{tabular}

Productivité et charge animale: La production fourragère calculée concerne la biomasse aérienne. Elle est exprimée en KgMS/ha ou en Tonnes MS/ha. Ainsi, la production fourragère brute du CSMB a été évaluée à 2,36 tMS/ha. Quant à la production de fourrage qualifié résultant de l'application de l'indice global de qualité
$(67,56 \%)$ à la production brute, a été de 2,02 tMS/ha. La capacité de charge globale est de 0,40 UBT/ha/an, soit 2,5ha/UBT/an. Le tableau 3 récapitule le bilan fourrager annuel du centre secondaire de multiplication de bétail (CSMB).

Tableau 3 : bilan fourrager annuel du CSMB.

\begin{tabular}{l|l}
\hline Paramètres globaux mesurés & Résultats obtenus \\
\hline Valeur pastorale brute $(\mathrm{Vpb})$ & $75,07 \%$ \\
Valeur pastorale nette $(\mathrm{Vpn})$ & 67,56 \\
Production de phytomasse $(\mathrm{Ph})$ & $2,36 \mathrm{tMS} / \mathrm{ha}$ \\
Production de fourrage qualifié (Pfq) & $2,02 \mathrm{tMS} / \mathrm{ha}$ \\
Capacité de charge (CC) & 0,40 UBT/ha/an \\
\hline
\end{tabular}

Individualisation des groupements pastoraux: L'influence des variables environnementales sur la distribution spatiale des espèces herbacées a été appréciée à travers l'analyse canonique des correspondances (ACC). L'analyse des résultats de l'ACC (matrice 56 relevés et 98 espèces) a permis d'isoler quatre grands ensembles nommés G1, G2, G3 et G4 (Figure 7) avec une inertie totale de 5,97 (tableau
4). Ces groupements sont mieux corrélés aux deux premiers axes d'ACC qui résument $43,3 \%$ de la variance totale. Ces axes sont également corrélés aux variables écologiques (texture du sol et géomorphologie). L'axe 1 est plus corrélé à la variable géomorphologie $(r=0,45$; $p=0,01$ ) et aux groupements $\mathrm{G} 1$ et $\mathrm{G} 2$. Cet axe exprime un gradient topographique, il est le plus explicatif de la variabilité floristique des communautés végétales.

Tableau 4 : Valeurs propres et pourcentage de la variance expliquée par les trois premiers axes de la CCA.

\begin{tabular}{l|l|l|l|lc}
\hline axes & $\mathbf{1}$ & $\mathbf{2}$ & $\mathbf{3}$ & Inertie totale P \\
\hline Valeurs propres & 0,244 & 0,190 & 0,139 & $\mathbf{5 , 9 7}$ & $\mathbf{0 , 0 1}$ \\
Corrélation espèces-environnement & 0,427 & 0,515 & 0,439 & & \\
Pourcentage cumulatif de variance expliquée & 4,1 & 7,3 & 9,5 & \\
Variance 'espèces-environnement' expliquée (\%) & 72 & 68,9 & 61,4 & \\
Somme de valeurs propres & & & $\mathbf{0 , 5 7 3}$ & \\
\hline
\end{tabular}




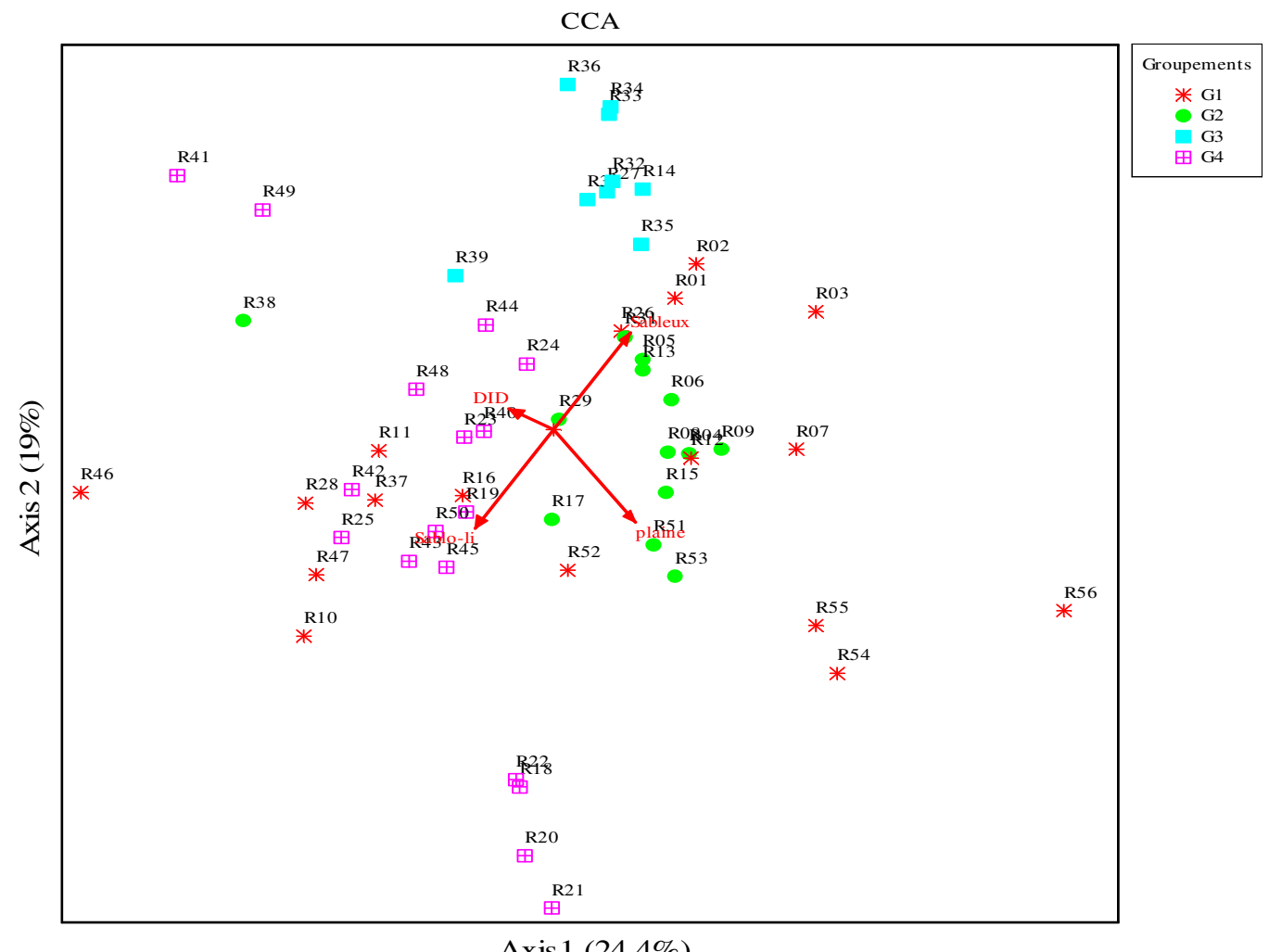

Figure 7 : analyse canonique des correspondances (CCA) entre les relevés et variables écologiques.

Quant à l'axe 2, il est mieux corrélé avec la variable texture $(r=0,50)$ et aux groupements $G 3$ et $G 4$, il traduit un gradient de la nature physique des sols.

Quatre groupements végétaux se sont différenciés, il s'agit de :

- Le groupement à Aristida mutabilis et Dactyloctenium aegyptium (G1) se développe sur tous les types de sol (sableux et sablo-limoneux) et unités géomorphologiques (plaine et dépression interdunaire) d'où la dispersion de ses relevés. Les espèces qui le composent sont indifférentes aux variables environnementales précitées. II est décrit à partir de 17 relevés et 79 espèces dont deux caractéristiques. Ce groupement est constitué majoritairement par des poacées avec un fourrage très apprécié par les bovins. II constitue pour ces derniers un excellent pâturage de la saison sèche.

- Le groupement G2 à Chloris prieurii et Spermacoce chaetocephala se localise sur un sol essentiellement sableux et sur la plaine. II est constitué de 13 relevés et 63 espèces dont deux caractéristiques, les autres sont des espèces compagnes. Ce groupement est également composé d'espèces graminéennes annuelles qui se conservent généralement très bien séchées sur pied durant toute la saison sèche et qui sont bien appréciées par les bovins. - Le groupement à Cenchrus biflorus et Fimbristilys hispidula (G3) est décrit à partir de 9 relevés et 55 espèces dont deux caractéristiques et 53 compagnes. Ce groupement est un excellent pâturage très bien apprécié par les bovins en toute saison de l'année. II évolue sur des sols sableux ayant une bonne aptitude pour retenir l'eau.

- Le groupement G4 à Tribulus terrestris et Corchorus tridens est formé de 17 relevés et 87 espèces dont 12 caractéristiques et 75 compagnes. II se distribue sur le sol sablo-limoneux et la dépression interdunaire. II est constitué essentiellement des légumineuses annuelles et représente le pâturage du début de la saison hivernale très utile aux bovins.

Les trois premiers pâturages sont constitués des plantes annuelles dont les pailles sèches sont très recherchées par les bovins durant toute l'année. En effet, leur conservation à l'état sec réussit bien.

Recouvrement du sol par la strate herbacée: Le recouvrement global du sol par les pâturages herbacés est de $86,5 \%$ et varie légèrement entre les groupements. Ce couvert herbacé a ainsi varié de $80 \%$ (G1) à $92 \%$ 
(G4). Le recouvrement spécifique moyen a été globalement faible. En effet, plus de $96 \%$ des espèces dénombrées ont présenté un recouvrement moyen inférieur à $10 \%$. Les espèces ayant les plus fortes contributions spécifiques demeurent celles qui possèdent le meilleur recouvrement spécifique.

Distribution spatiale des groupements pastoraux : Les degrés d'homogénéité spatiale et interne d'espèces herbacés ont été appréciés par l'emploi des indices de diversité et de régularité. L'indice global de diversité $\left(\mathrm{H}^{\prime}\right)$ est de 4,7 bits et l'équitabilité de Piélou (E) 0,7 bits avec Hmax 6,9 espèces (tableau 5). Cette diversité spécifique a varié selon les groupements pâturés.
L'indice de diversité ( $\left.H^{\prime}\right)$ a fluctué d'un minimum de 2,7 bits en $\mathrm{G} 3$ et à un maximum de 4,4 bits en $\mathrm{G} 1$ et l'indice l'équitabilité de Piélou a suivi la même tendance. Les valeurs obtenues à travers le calcul de l'indice de similitude de Jaccard pour les pâturages comparés deux à deux restent supérieures ou égales à $50 \%$ (Tableau 6). Cet indice qui traduit la similitude entre les groupements végétaux pâturés a varié entre $50 \%$ (moyenne diversité ß) et $60 \%$ (G1 et G4). Cette meilleure similitude entre $\mathrm{G} 1$ et $\mathrm{G} 4(60 \%)$ indique qu'ils sont les plus homogènes, ils partagent moyennement le même cortège floristique.

Tableau 5 : indices de diversité et de régularité.

\begin{tabular}{l|l|l|l|l|l}
\hline Indices & Global & G1 & G2 & G3 & G4 \\
\hline Richesse spécifique $(\mathrm{S})$ & 119 & 79 & 63 & 55 & 87 \\
\hline Indice de Shannon $\left(\mathrm{H}^{\prime}\right)$ & 4,7 & 4,4 & 3,8 & 2,7 & 4,2 \\
\hline Equitabilité de Pielou $(\mathrm{E})$ & 0,7 & 0,7 & 0,6 & 0,4 & 0,6 \\
\hline Diversité maximale $\left(\mathrm{H}^{\prime}\right.$ max) & 6,9 & 6,3 & 6 & 5,8 & 6,4 \\
\hline
\end{tabular}

Tableau 6 : degré de communauté végétale les groupements comparés deux à deux

\begin{tabular}{|l|l|l|l|l|}
\hline Groupements comparés & G1 & G2 & G3 & G4 \\
\hline G1 & $100 \%$ & & & \\
\hline G2 & 50 & $100 \%$ & & \\
\hline G3 & 50 & 50 & $100 \%$ & \\
\hline G4 & 60 & 50 & 50 & $100 \%$ \\
\hline
\end{tabular}

Valeur pastorale des groupements pastoraux: L'analyse du spectre fourrager (figure 8) montre une forte dominance de la catégorie d'espèces de bonne valeur pastorale (Bvp) pour l'ensemble des groupements pâturés avec des proportions variant de $43,25 \%$ en G4 à $83,31 \%$ en $\mathrm{G} 2$. Elles sont suivies par les espèces de faible valeur pastorale (Fvp). Cette forte dominance se justifie par les contributions spécifiques élevées des bonnes graminées suivantes : C. biflorus (Csi $=16,77 \%$ et IS $=3$ ), C. prieurii (Csi $=13,69 \%$ et IS $=3$ ), $A$. mutabilis (Csi $=11,09 \%$ et IS $=3$ ) et Schoenefeldia gracilis Kunth. (Csi $=6,69 \%$ et IS $=3$ ). Au sein des groupements, les valeurs pastorales brutes et nettes (Tableau 7 ) les plus importantes sont lues au niveau du groupement G2 $(\mathrm{Vpb}=88 \%$ et $\mathrm{Vpn}=74 \%)$. Ces valeurs permettent de déduire que ce pâturage est excellent. Par ailleurs, on relève que toutes les valeurs pastorales brute et nette sont supérieures à $50 \%$.

Phytomasse et charge animale en fonction des groupements : La production de la matière sèche varie selon le groupement (Tableau 6). Le groupement G2 a été le plus productif (1,37 KgMS/ha) et la valeur de la phytomasse la plus faible a été observée en G4 $(0,69$ $\mathrm{KgMS} / \mathrm{ha}$ ). L'application de la valeur pastorale pondérée à la biomasse épigée a permis de qualifier le fourrage produit. Ainsi, ces valeurs ci-dessus deviennent respectivement $1,01 \mathrm{KgMS} / \mathrm{ha}$ et $0,38 \mathrm{KgMS} / \mathrm{ha}$ de fourrage qualifié. Les capacités de charge théorique ont varié selon les groupements (Tableau 6). Ainsi, la capacité de charge la plus importante a été notée au niveau du groupement G2 $(0,20 \mathrm{UBT} / \mathrm{ha} / \mathrm{an})$, soit 3,7 ha/UBT/an et la plus faible au niveau de G4 $(0,14$ UBT/ha/an), soit 7,14 ha/UBT/an au pic de phytomasse herbacée. D'une manière générale, les capacités de charge du centre ont été faibles. L'analyse du spectre de production fourragère par espèce herbacée (Figure 9) relève que ce sont les espèces $A$. mutabilis $(214,90 \mathrm{~kg}$ MS/ha), C. biflorus $(194,88 \mathrm{~kg} \mathrm{MS} / \mathrm{ha})$, C. prieurii (186,90 kg MS/ha), A. ovalifolius (80 kg MS /ha), qui sont les plus productives. On remarque aussi que les espèces les plus productives sont des espèces de bonne valeur pastorale (Bvp). 
Idrissa et al., J. Appl. Biosci. 2020 Caractérisation des pâturages herbacés sahéliens à accès limité : Cas du Centre Secondaire de Multiplication de Bétail de Sayam (Diffa).

Tableau 7 : paramètres d'appréciation de fourrage produit et capacité de charge.

\begin{tabular}{|l|l|l|l|l|}
\hline Paramètres mesurés en fonction des groupements & G1 & G2 & G3 & G4 \\
\hline Recouvrement global (\%) & 80 & 84 & 90 & 92 \\
\hline Valeur pastorale brute (\%) & 77,81 & 87,99 & 80,68 & 59,73 \\
\hline Valeur pastorale nette (\%) & 62,25 & 73,91 & 72,26 & 54,73 \\
\hline Production (tMS/ha) & 1,02 & 1,37 & 1,26 & 0,69 \\
\hline Fourrage qualifié (tMS/ha) & 0,63 & 1,01 & 0,92 & 0,38 \\
\hline Capacité de charge (UBT/ha/an) & 0,20 & 0,27 & 0,25 & 0,14 \\
\hline Nombre d'hectares pour chaque UBT (ha/UBT/an) & 5 & 3,7 & 4 & 7,14 \\
\hline
\end{tabular}

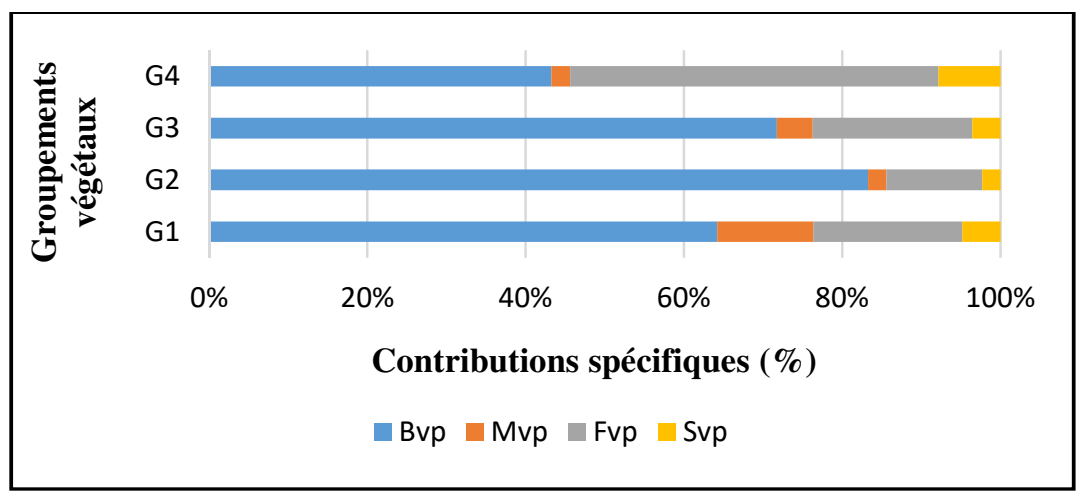

Figure 8 : spectre des catégories fourragères en fonction des groupements.

Bvp : bonne valeur pastorale, Mvp : moyenne valeur pastorale, Fvp : faible valeur pastorale, Svp : sans valeur pastorale.

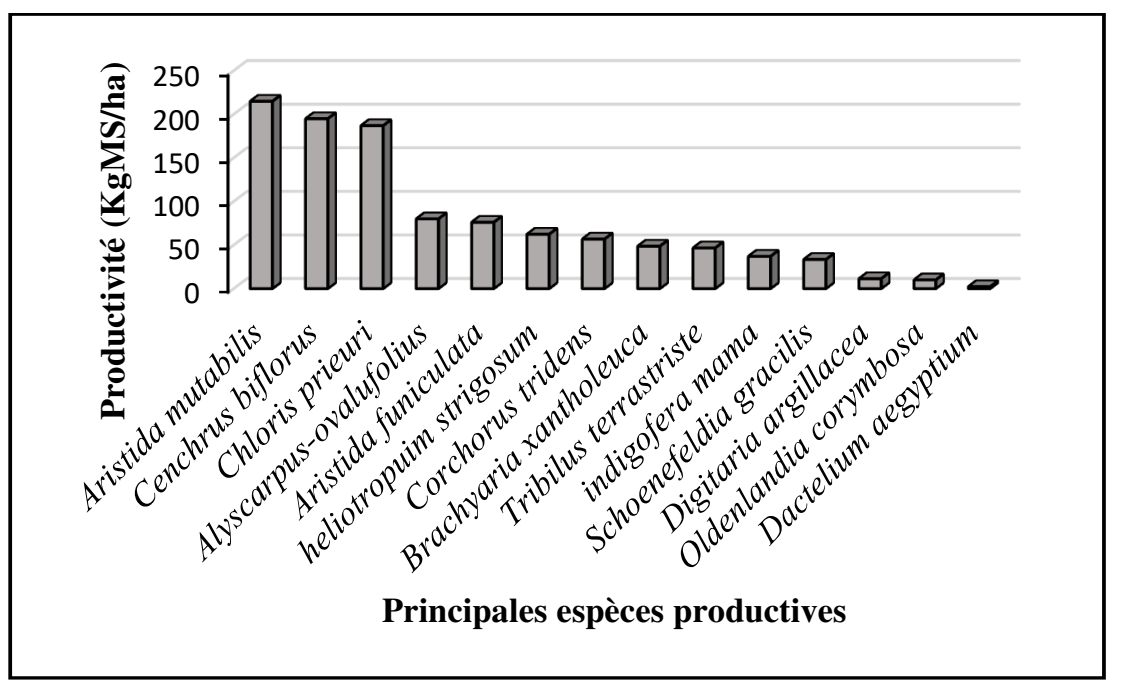

Figure 9 : productivité selon les principales espèces.

\section{DISCUSSION}

Le Centre Secondaire de Multiplication de Bétail de Sayam est riche de 98 espèces herbacées réparties dans 59 genres et 27 familles botaniques. Cette richesse spécifique est variable entre les groupements, elle est plus élevée au niveau du G4 et plus faible dans le G3. La flore herbacée du CSMB est donc plus riche dans la partie moins protégée (périphérique) par rapport au centre plus protégé. Cette différence peut être due à la position topographique, à la nature du sol et surtout aux activités anthropiques notamment le surpâturage dont fait l'objet cette partie. Ces résultats sont similaires à ceux obtenus par Saidou et al. (2013) à des latitudes 
similaires (station de Toukounous). Ces auteurs ont recensé 75 espèces herbacées. La différence floristique observée entre les deux stations serait liée à l'effort de protection. En effet, une bonne partie du périmètre de la station de Sayam n'a plus de clôture grillagée. Cette situation facilite l'accès aux pâturages des troupeaux des bovins et camelins. Ces derniers exercent une forte pression sur les ressources fourragères disponibles. II en résulte un surpâturage qui déclenche une dégradation des parcours (Coulibaly et al., 2009). Celleci se traduit par une augmentation de la biodiversité taxonomique (Sawadogo et al., 2012, Botoni et al., 2006). Selon Burrel et Baudry (2003) jusqu'à un certain seuil, les perturbations augmentent la diversité d'un écosystème. Par ailleurs, le feu de brousse ayant ravagé en 2016 une large bande (dizaine de km) du CSMB, l'année suivante, on a assisté à une pousse massive des phorbes sur la bande parcourue par le feu. Cela peut influencer le nombre d'espèces. Cette richesse spécifique en herbacées peut aussi être reliée à l'abondance de la pluviométrie. En effet, 2016 et 2017 ont enregistré des fortes précipitations bien réparties dans le temps et dans l'espace. Les familles botaniques ont été largement dominées par les Poaceae. Ce constat a été fait par plusieurs auteurs ayant conduit des travaux phytosociologiques dans la bande soudano- sahélienne (Kaou et al., 2017, Saidou et al., 2013, Soumana, 2011, Morou, 2010, Mahamane, 2005, Akpo et al., 2002, Saadou, 1990). La prédominance de cette famille témoigne de sa résistance aux différentes perturbations environnementales qui se produisent au sahel, en développant des stratégies diverses lui permettant de se maintenir et d'évoluer normalement (Breman et De Ridder, 1991). Elles sont caractéristiques des régions sahéliennes. Cela confirme l'appartenance du site à la zone sahélienne caractérisée par une courte saison humide avec des précipitions courtes et souvent violentes (Bodart et al., 2010). L'abondance et dominance des thérophytes signifient qu'ils s'adaptent mieux aux conditions pédoclimatiques de la zone. Cette adaptation est rendue possible par ce que les thérophytes bouclent leur cycle développement durant la saison pluvieuse et passent à l'état de graine pendant la période défavorable (Morou, 2010). Les parties végétatives se détruisent par dessiccation (Mamadou et al., 2018). La prépondérance des thérophytes reflète avec rigueur l'aridité climatique de la zone. L'indice de cette aridité est la forte présence d'espèces caractéristiques des régions climatiques sèches: $A$. mutabilis, A. ovalifolius, $S$. gracilis ...etc. Les types phytogéographiques sont des bons indicateurs $d u$ dynamisme ou de la stabilité des communautés végétales (Sinsin, 1993). L'examen des spectres bruts et pondérés des types chorologiques montre une dominance absolue des espèces à large distribution. Le même auteur indique que la forte proportion d'espèces à large distribution est un indice de perturbation et signale que la flore perd de sa spécificité. Une appréciation globale des groupements peut se faire en tenant compte de leur recouvrement, leur biomasse, la valeur pastorale de leur fourrage et la capacité de charge de chacun. Ils ont été abordés selon l'ordre de leur distinction.

Le groupement à Aristida mutabilis et Dactyloctenium aegyptium (G1), est établi à partir de 17 relevés et 79 espèces herbacée. Parmi lesquelles figurent deux espèces caractéristiques portant le nom du groupement avec des recouvrements spécifiques moyens respectifs de $15,06 \%$ et $6,15 \%$. Le recouvrement spécifique demeure toujours faible. En effet, parmi ces espèces seules 3 ont un recouvrement spécifique moyen supérieur à $10 \%$. En revanche, le recouvrement global du groupement est élevé $(80 \%)$ avec une distribution spatiale régulière sur l'ensemble de la station. Saidou et al. (2010) ont fait le même constat au niveau de la station expérimentale de Toukounous. Ce pâturage dominé par $A$. mutabilis est formé d'espèces moins exigeantes en eau et indifférentes aux variables écologiques, c'est-à-dire qu'elles se distribuent sur l'ensemble des unités paysagères pastorales et des types de sol. Ces résultats corroborent à ceux trouvés par Saidou et al. (2013) à la station sahélienne expérimentale de Toukounous, où ils ont indiqué que $A$. mutabilis et $D$. aegyptium font partie du lot d'espèces moins exigeantes en eau et à la texture du sol. Ce groupement constitue un excellent pâturage de saison sèche. En effet, il est composé des graminées annuelles dont les pailles sèches très fines, sont recherchées par les bovins, d'où l'attractivité de la zone d'étude pendant cette saison. La valeur pastorale brute du groupement est de $77,81 \%$, ce qui confirme la qualité pastorale de ce pâturage. Ce résultat est comparable à celui trouvé par Saidou et al. (2010) dans un écosystème similaire au nord-est de Niamey (Toukounous). La valeur de cet indice reste dépendante de la composition spécifique du spectre fourrager (Ngom, 2012). Si les espèces de bonne valeur pastorale et moyenne valeur pastorale prédominent un pâturage, alors l'indice global de qualité est plus élevé. En analysant le spectre des catégories fourragères, on observe une large prépondérance des espèces de bonne valeur pastorale (plus de $65 \%$ de Csi totale, Is $=3$ ). Cette formation à Aristideae présente un 
rendement moyen en matière sèche de 1,02 tMS/ha avec une charge animale de 0,20 UBT/ha/an soit 5 ha/UBT/an. Cette faible capacité de charge est due à l'application de l'indice global de qualité (IGQ) qui fiabilise son estimation (Barral et al., 1983). La valeur de cet indice a été estimée à $62,25 \%$, cela signifie que la proportion de la phytomasse herbacée brute absorbée par les bovins est de 62,25\%. Cependant, cette formation à Aristideae est la plus affectée négativement par les feux de brousse en raison de sa composition floristique (graminées annuelles à pailles sèches fines très inflammables).

Le groupement à Chloris prieurii et Spermacoce chaetocephala (G2) est constitué de 13 relevés et 66 espèces avec une couverture herbacée spécifique de $21,58 \%$ et $0,08 \%$ respectivement. Ce groupement dominé par Chloris prieurii a un recouvrement global de $84 \%$. Cette espèce est la deuxième en termes de recouvrement et contribution spécifique au tapis herbacé de la station avec une répartition spatiale assez régulière. Le pâturage de ce groupement figure aussi parmi les meilleurs pâturages de la saison sèche. En effet, il se caractérise par des Poaceae de forte appétence en état sec et qui sont bien affourragées par le bétail notamment les bovins en cet état. La composition floristique est moyennement diversifiée avec l'indice de Shannon ( $H^{\prime}$ ) qui est de 3,38 bits et l'indice d'équitabilté de Piélou suit la même la tendance. Ces résultats rejoignent ceux obtenus par Saidou et al. (2013) dans la station sahélienne de Toukounous. Cette similitude entre ces deux milieux s'expliquerait par le fait qu'ils partagent les mêmes conditions pédoclimatiques. Les valeurs pastorales brutes et nettes sont respectivement de $88 \%$ et $73,91 \%$. Ces valeurs indiquent un pâturage herbacé présentant un intérêt pastoral indéniable. Elles attestent que les espèces de bonne valeur pastorale $(\mathrm{ls}=3$ ) prédominent la végétation herbacée du groupement. L'analyse $\mathrm{du}$ spectre fourrager le confirme, car ces espèces ont participé pour plus de $80 \%$ à la formation de l'indice global de qualité. Ces résultats sont similaires à ceux trouvés par Saidou et al. (2010) à Toukounous. En revanche, ils sont radicalement opposés à ceux obtenus par Alhassane et al. (2018) sur les parcours de la région de Maradi où les espèces de bonne valeur pastorale sont les plus rares $(8 \%)$. Cette différence serait liée d'une part à l'effort de protection dont bénéficie le centre comparativement aux parcours et d'autre part les espèces de bonne valeur pastorale deviennent rares dans une formation pastorale à forte pression animale. En effet, les animaux aux pâturages choisissent les espèces de bonne valeur pastorale et moyenne valeur pastorale au détriment d'autres catégories fourragères. D'où la rareté de cette catégorie. Le rendement en sec est de 1,37 tMS/ha de paille. Ce pâturage est le plus productif de la station. Cela se justifie par le fait qu'il se localise dans l'aire protégée de la station qui bénéficie plus de surveillance par rapport aux parties les plus externes. Ces dernières faisaient l'objet d'une incursion régulière des animaux transhumants et locaux. La capacité de charge suit la même dynamique bien qu'elle soit globalement faible $(0,27$ UBT/ha/a soit 3,7 ha/UBT/an. Saidou et al. (2010) ont trouvé 3,5 ha/UBT/an à Toukounouss.

Le groupement à Cenchrus biflorus et Fimbristilys hispidula (G3) est décrit à partir de 9 relevés avec une diversité systématique de 55 espèces dont 53 sont compagnes. Cenchrus biflorus et Fimbristilys hispidula sont les espèces caractéristiques de ce pâturage avec des recouvrements spécifiques moyens de $22 \%$ et $0,53 \%$ respectivement. $\bar{A}$ l'échelle du centre, Cenchrus biflorus est le plus recouvrant $(15,25 \%)$ et détient le score le plus élevé quant à la contribution spécifique $(16,77 \%)$ au sein du tapis herbacé. Ce groupement végétal se développe sur des sols sableux ayant une bonne capacité de rétention en eau et colonise essentiellement les dépressions inter-dunaires. $\mathrm{Ce}$ pâturage est moins soumis à la pression pastorale, car il se localise dans la zone centrale. Cette dernière n'est pas exploitée par les animaux extérieurs, car bénéficiant d'effort de protection de la part de l'administration du CSMB. Ce pâturage est le plus apprécié des bovins durant toutes les saisons de l'année selon les bergers enquêtés, puis qu'il est composé majoritairement d'espèces très bien appétées en sec. II s'agit notamment de $S$. gracilis, Aristida funiculata. II constitue un apport alimentaire précieux pour les bovins pendant la période de sèche. II représente cependant le pâturage le moins diversifié avec 2,7 et 0,4 bits respectivement pour les indices de diversité $\left(H^{\prime}\right)$ et de régularité $(E)$. Cela est dû à l'effort de protection. Des observations analogues ont été faites par plusieurs auteurs Ouest africains ayant effectués des travaux phytosociologiques la zone soudano-sahélienne notamment dans le parc W (Sawadogo et al., 2012); Mahamane, 2005). Ces auteurs ont montré que le noyau central est moins diversifié comparativement aux deux autres zones (transition et périphérie). Ils attribuent cette différence aux activités anthropiques (pression pastoral et agricole) qui augmentent la richesse floristique d'une formation végétale. La qualité des ressources herbagères du groupement est prouvée par la valeur pastorale très 
élevé $(80,68 \%)$. Cette valeur permet de déduire que ce pâturage présente un intérêt pastoral de haute qualité. Cette dernière est à relier à la forte représentation des espèces de bonne valeur pastorale (ls = 3). L'examen du spectre fourrager montre qu'elles participent à plus $70 \%$ des Csi globales. La production fourragère brute du groupement est relativement élevée (1,26 tMS/ha) avec une capacité de charge de 0,25 UBT/ha/an soit 4 ha/UBT/an

\section{Le groupement G4 à Tribulus terrestris et Corchorus} tridens est formé à partir de 17 relevés et 87 espèces végétales dont 12 caractéristiques. Le recouvrement global de ce groupement est le plus important de l'ensemble des pâturages identifiés (92\%). Les espèces caractéristiques portant le nom du groupement possèdent des recouvrements spécifiques moyens respectifs de $23,78 \%$ et $6,71 \%$. Ce groupement est le domaine des phorbes qui constituent un bon pâturage du début de la saison pluvieuse mais qui disparaissent rapidement. L'espèce Corchorus tridens a littéralement colonisé la bande parcourue par les feux de brousse de 2016. L'une des conséquences immédiates du passage de feu est l'amorce de la recomposition floristique du tapis herbacé de cette portion qui était couverte par les graminées annuelles. Ce tapis herbacé a été remplacé brusquement par les phorbes. $\mathrm{Ce}$ groupement représente les parties où la pression pastorale est forte. $\mathrm{Ce}$ pâturage est le plus riche floristiquement. Cette richesse en espèces herbacées serait liée à la forte exploitation de ce pâturage. Un constat similaire a été fait par Botoni et al., 2006 dans l'Ouest Burkinabé. La forte pression pastorale que connait cette formation à phorbes se justifie par sa proximité aux hameaux riverains dont les animaux s'y introduisent fréquemment pour paître. Cette forte exploitation pastorale a appauvri

\section{CONCLUSION}

Le présent travail a permis de faire un état des lieux sur la diversité de la flore herbacée du CSMB de Sayam. Cette diversité est relativement élevée comparée à celle de Toukounous et varie selon les pâturages. Elle est plus élevée à la périphérie et faible au centre de la station. Du point de vue pastoral, les espèces de bonne qualité pastorale représentent la proportion des catégories fourragères la plus importante (Bvp) et se concentrent plus dans la zone centrale mieux protégée et celles peu ou pas appétées se distribuent essentiellement dans les parties à forte pression pastorale. Le front de colonisation d'espèces délaissées ou faiblement appétées remonte progressivement sous l'effet de ce pâturage en espèces de bonne qualité pastorale. II est plutôt riche en espèces herbagères invasives nitrophiles de qualité pastorale allant de faible à nulle. II s'agit notamment de Tribulus terrestris, Heliotropuim strigosum, Amaranthus spinosus, Corchorus tridens, Cyperus amabilis, Fimbristilys hispidula, Gynandropsis gynandra, Aerva javanica. En effet, ces espèces portent l'indices spécifique faible ou nul. L'abondance de ces espèces confirme que ce milieu est le plus pâturé. Les valeurs pastorales brutes et nettes de ce pâturage demeurent les plus faibles de l'ensemble de pâturages et sont respectivement de $59,73 \%$ et $54,73 \%$. Ce pâturage est nutritivement de moyenne qualité, car selon Daget et Godro (1995) un pâturage est considéré de bonne qualité lors que sa valeur pastorale atteint au moins $65 \%$. Ces faibles valeurs observées sont naturellement liées à la qualité de la composition floristique dominée par des espèces non ou peu appétées. Selon Sawadogo et al. (2012) les activités pastorales et agricoles induisent une baisse de la valeur pastorale des parcours, car celle-ci dépend tout à la fois de la quantité de la phytomasse herbacée et de sa qualité. Ce groupement est le moins productif avec 0,69 tMS/ha/an. Cette faible production serait liée à la nature du sol. En effet une bonne partie des relevés de ce groupement se localise sur un sol squelettique à faible productivité. II détient également la plus faible capacité de charge de l'ordre 0,14 UBT/ha/an soit 7,14 ha/UBT/an. La capacité de charge étant liée à la productivité, c'est tout à fait normal qu'elle soit trop faible car la productivité de ce milieu étant aussi faible. L'intérêt pastoral de ce pâturage est limité car étant formé globalement d'espèces à faible valeur pastorale qui disparaissent à la fin saison pluvieuse laissant le sol nu. Cette situation favorise la progression du front dunaire qui envahit particulièrement la strate herbacée.

pâture vers le noyau riche en espèces productives de haute qualité pastorale. Les activités anthropiques notamment la forte exploitation pastorale peut déclencher une recomposition de la strate herbacée. Celle-ci se manifeste déjà au niveau de la bande parcourue par le feu dont le tapis herbacé est dominé par des phorbes. D'où l'urgence de stopper ce front en clôturant l'intégralité du périmètre de la station. Cela permettra d'éviter de provoquer des déséquilibres écologiques préjudiciables à la survie des nombreuses espèces spécifiques à cet écosystème pastoral particulier. 


\section{RÉMERCIEMENTS}

Les auteurs de cet article remercient sincèrement les responsables du CSMB pour leur disponibilité, leur amabilité et leur soutien en logistique lors de la collecte

\section{RÉFÉRENCES}

A, A. (1983). Contribution à l'étude des écosystèmes steppiques du sud-oranais. Phytomasse, productivité et application pastorale. Thèse Doct., USTHB, Alger. 142p.

Agonyissa D, S. B. (1998). Productivité et capacité de charge des pâturages naturels au Bénin. Revue Élev. Méd. Vét. Pays Trop., 51(3) : 239-246.

Akpo LE, D. M. (2002). Durée de jachère et valeur pastorale de la végétation herbacée en zone soudanienne au Sénégal. Révue Elev. Méd. Vét. Pays Trop, 55(4) : 275-283.

Alhassane A, I. S. (2018). Productivité, valeur pastorale et capcité de charge des parcours naturels de la région de Maradi, Niger. Int. J. Biol. Chem. Sci. 12(4): 1705-1716, doi : 10.4314/ijbcs.v12i4.15.

Alhassane, A. (2019). Typologie, valeur pastorale, productivité et capacité de charge des pâturages naturels au Centre-Sud du Niger suivant le gradient climatique Sud-Nord. Thèse Doct., Université Dan Dicko Dankoulodo de Maradi. Niger. 179p.

Barma, S. L. (2017). Contribution à l'étude des parasitoses chez les vaches kouri dans le centre secondaire de multiplication de bétail de SAYAME. Thèse Doct., de médecine vétérinaire, Université Cheick Anta Diop de Dakar, Sénégal. 173p.

Barral H., E. B. (1983). Systèmes de production d'élevage au Sénégal dans la région du férlo. Synthèse de fin d'études d'une équipe de recherche pluridisciplinaire. ACC/RIZAT (LAT), GERDAT- Orstom, 172p.

Bodart C, A. O. (2010). Suivi de l'activité des dunes au Niger au moyen de la cohérence interférométrique. BSGLg, 1 (54). ERS $1 / 2$.

Botoni Liehoun E., P. D. (2006). Activités de pâturage, biodiversité et végétation pastorale dans la zone Ouest du Burkina Faso. Revue Elev. Méd. Vét. Pays trop., 59 (1-4) : 31-38.

Breman H., e. N. (1991). Manuel sur les Pâturages des Pays Sahéliens. Karthala, ACCT, CABO-DLO et CTA; 485p.

CIRAD. (1996). Atlas du bassin Lac Tchad. CTA, 158p. de données, soutien sans lequel ce travail n'aurait pas été possible. Ces remerciements vont également à l'endroit des relecteurs anonymes du manuscrit.

Coulibaly D, R. P.-C. (2009). Dynamiques territoriales et changements des modes de gestion des ressources pastorales au Mali Sud (Mali). Renc. Rech. Ruminants : 357-360.

Daget P, J. P. (1990). Notion de valeur pastorale. Institut Botanique, Montpellier R et re 3 (1) : 5-8.

Daget P, P. J. (1971.). Une méthode d'analyse phytologique des prairies, Critères d'application. Ann. Agron., 22 (5-41).

Daget, p. e. (1995). Pastoralisme: Troupeaux, espaces et sociétés. hatier, AUPELF, UREF, Universités francophones.

Douma S, S. O. (2010). Analyse du peuplement herbacé de la station sahélienne expérimentale de Toukounous (Niger) : Composition floristique et valeur pastorale. Revue Sécheresse 2 (21) : 154- 60, doi : 10. 1684/sec. 2010.0243.

Gérard Chouquer, «. F. (2003). Écologie du paysage : Concepts, méthodes et applications ",. Études rurales [En ligne], 167-168 |, mis en ligne le 17 décembre 2004, consulté le 14 juillet 2020. URL

http://journals.openedition.org/etudesrurales/2 968.

Grouzis, A. L. (2000). Valeur pastorale des herbages en région soudanienne, le cas des parcours sahéliens du Nord- Sénégal. Tropicultura, 18 (1) : 1-8.

I, S. (2011). Groupements végétaux pâturés des parcours de la région de Zinder et Stratégies développées par les éleveurs Uda'en. Thèse Doct, Université Abdou Moumouni de Niamey, Niger, 234p.

Issa S, J. d. (2012). État des ressources pastorales dans une terre d'accueil et de transit des pasteurs transhumants : le terroir de Kotchari (sud- est du BurKina Faso). Rev. Écol. (Terre Vie), vol. $67: 157-178$.

J, B. (1967). Flore Sénégal (2e éd). Edition Clair Afrique : Dakar, Sénégal, 485p.

K. I. Adamou, H. M. (2018). Étude rétrospective des paramètres de reproduction du taurin de Kouri à la station de Sayam au Niger. TROPICULTURA 36 (1) : 87-98. 
Kiari A. K. K, O. L. (2017). Diversité floristique et structure de la végétation dans la zone dunaire du sud-est du Niger : Cas de Mainé soroa. Journal of Applied Biosciences 120: 1205312066 : https//dx.doi.org/10.4314/jab.v120i1.8.

M, B. (1997). L'Agroforesterie pour les Productions Animales. Centre Technique de Coopération Agricole et Rurale - Centre Internationale pour la Recherche en Agroforesterie. 384p.

Mahamane, A. (2005). Études floristique, phytosociologique et phytogéographique de la végétation du Parc Régional du $W$ du Niger. Thèse Doct., Université Libre de Bruxelles. $484 p$.

Mamadou A. J., S. D. (2018). Analyse De L'état De La Diversité Floristique Des Plateaux Suivant Un Gradient D'aridité Nord- Sud Dans La Réserve De Biosphère Du W Du Niger. European Scientific Journal. Vol.14, No.3 : 156-178.

Morou B. (2010). Impact de l'occupation des sols sur l'habitat de la girafe au Niger et enjeux pour la sauvegarde du dernier troupeau de girafes de l'Afrique de l'Ouest. Thèse de doctorat en Biologie appliquée, Faculté des Sciences et Techniques, Université Abdou Moumouni de Niamey. 198p.

Ngom D, A. B. (2012). Qualité pastorale des ressources hérbagères de la réserve de biosphère du Férlo (Nord- Sénégal). Int. J. Biol. Chem. Sci. 6(1) : 186-201,

doi : //dx.doi.org/10.4314/ijbcs.v6i1.17.

NIGER, R. D. (2013). Stratégie de développement durable de l'élevage (SDDEL 2013-2035). 78p.

P, P. (1999). Les Poaceae du Niger. Boissiera 56 (1), $766 \mathrm{p}$.

R., D. (1960). Critères de jugement de la valeur agronomique des prairies. Fourrages 4 (1) :8398.

Rhissa, Z. (2010). Revue du secteur de l'élevage au Niger. Rapport réalisé par FAO/SFW. 115p.

Saadou, M. (1990). La végétation des milieux drainés à l'Est du fleuve Niger. Thèse Doct., Université Abdou Moumouni de Niamey, Niger, 539.

Saidou OUSSEINA, R. F. (2013). Dynamique du peuplement herbacé de la station sahélienne expérimentale de Toukounous (Filingué Niger). Int. J. Biol. Chem. Sci. 7(2) : 657-671. DOI : http://dx.doi.org/10.4314/ijbcs.v7i2.22.

Sinsin B. (1993). Phytosociologie, écologie, valeur pastorale, production et capacité de charge des pâturages naturels du périmètre Nikki-Kalalé au
Nord-Bénin. Thèse Doct., Université Libre de Bruxelles. 390p.

Yoka J, J. L. (2010). Influence du sol sur la répartion et la réproduction de la phytomasse des savanes de la cuvette Congolaise. Géo-Eco- Trop 34(1) : 63-74. 